\title{
Extended warm gas in the ULIRG Mrk273: Galactic outflows and tidal debris
}

\author{
J. Rodríguez Zaurín ${ }^{1,2}$, C. N. Tadhunter ${ }^{3}$, D. S. N. Rupke ${ }^{4}$, S. Veilleux ${ }^{5}$, H. W. W. Spoon ${ }^{6}$, M. Chiaberge ${ }^{7}$, \\ C. Ramos Almeida ${ }^{1,2}$, D. Batcheldor ${ }^{8}$, and W. B. Sparks ${ }^{7}$
} 1 Instituto de Astrofísica de Canarias, 38205 La Laguna, Tenerife, Spain
2 Departamento de Astrofísica, Universidad de La Laguna, 438205 Tenerife, Spain
3 Department of Physics and Astronomy, University of Sheffield, Sheffield S3 7RH, UK
e-mail: c.tadhunter@sheffield.ac.uk
4 Department of Physics, Rhodes College, Memphis, TN 38112, USA
5 Department of Astronomy, University of Maryland, College Park, MD 20742, USA
6 Cornell University, CRSR, Space Sciences Building, Ithaca, NY 14853, USA
7 Space Telescope Science Institute, 3700 San Martin Drive, Baltimore, MD 21218, USA
8 Physics and Space Sciences Department, Florida Institute of Technology, 150 West University Boulevard, Melbourne,
FL 32901, USA

Received 30 January 2014 / Accepted 23 July 2014

\section{ABSTRACT}

\begin{abstract}
We present new HST/ACS medium- and narrow-band images and optical Isaac Newton Telescope long-slit spectra of the merging system Mrk273. The HST observations sample the [OIII] $\lambda \lambda 4959,5007$ emission from the galaxy and the nearby continuum. These data were taken as a part of a larger study of ultraluminous infrared galaxies (ULIRGs) with the aim of investigating the importance of the warm, AGN induced outflows in such objects. The HST images show that the morphologies of the extended continuum and the ionised gas emission from the galaxy are decoupled, extending almost perpendicular to each other. In particular, we detect for the first time a spectacular structure of ionised gas in the form of filaments and clumps that extend $\sim 23 \mathrm{kpc}$ to the east of the nuclear region. The quiescent ionised gas kinematics at these locations suggests that these filaments are tidal debris left over from a secondary merger event that are illuminated by an AGN in the nuclear regions. The images also reveal a complex morphology in the nuclear region of the galaxy for both the continuum and the [OIII] emission. Consistent with this complexity, we find a wide diversity of emission line profiles in these regions. Kinematic disturbance in the form of broad $\left(F W H M>500 \mathrm{~km} \mathrm{~s}^{-1}\right)$ and/or strongly shifted $\left(|\Delta V|>150 \mathrm{~km} \mathrm{~s}^{-1}\right)$ emission line components is found at almost all locations in the nuclear regions, but confined to a radius of $\sim 4 \mathrm{kpc}$ to the east and west of the northern nucleus. In most cases, we are able to fit the profiles of all the emission lines of different ionisation with a kinematic model using two or three Gaussian components. From these fits, we derive diagnostic line ratios that are used to investigate the ionisation mechanisms at the different locations in the galaxy. We show that these line ratios are generally consistent with photoionisation by an AGN as the main ionisation mechanism. Finally, the highest surface brightness [OIII] emission is found in a compact region that is coincident with the so-called SE nuclear component. The compactness, kinematics, and emission line ratios of this component suggest that it is a separate nucleus with its own AGN. At this stage, further observations are required to confirm the dual (or multiple?) AGN nature of Mrk273.
\end{abstract}

Key words. ISM: kinematics and dynamics - ISM: jets and outflows - galaxies: interactions - galaxies: active - galaxies: evolution

\section{Introduction}

Fast outflows induced by the central active galactic nucleus (AGN) are now almost invariably detected in dusty mergers with Seyfert-like optical nuclei (e.g. Westmoquette et al. 2012; Rodríguez Zaurín et al. 2013; Veilleux et al. 2013; Rupke \& Veilleux 2013b). These outflows are a key element in simulations of galaxy evolution through major mergers, where they are used to regulate the correlation between the black hole mass and the host galaxy properties (e.g. Silk \& Rees 1998; Fabian 1999; Benson et al. 2003; Johansson et al. 2009). In particular, they are predicted to be extremely powerful towards the final stages of the merger, when the merging nuclei coalesce, potentially quenching the surrounding star formation activity (e.g. di Matteo et al. 2005; Springel et al. 2005; Johansson et al. 2009; Hopkins \& Elvis 2010). However, from an observational perspective, the nature of their interaction with the ISM in the host galaxies and importance relative to the outflows induced by star formation activity have yet to be firmly established.

In this context, ultra luminous infrared galaxies (ULIRGs, $L_{\mathrm{IR}}>10^{12} L_{\odot}$ ) are ideal objects to investigate the physical processes involved in AGN-induced outflows. Their prodigious far-IR radiation represents the dust re-processed light of nuclear power sources (starbursts and/or AGN). In addition, ULIRGs almost invariably show morphological evidence consistent with triggering of the activity in major galaxy mergers (e.g. Murphy et al. 1996; Clements et al. 1996; Sanders \& Mirabel 1996; Surace et al. 2000; Surace \& Sanders 2000; Scoville et al. 2000; Veilleux et al. 2002). Therefore, ULIRGs represent just the situation modelled in many of the most recent hydrodynamic simulations.

As expected from the merger simulations, AGN-induced outflows in ULIRGs have recently been found in all gas phases: neutral (Rupke \& Veilleux 2011, 2013b), ionised (e.g. 
Westmoquette et al. 2012; Rodríguez Zaurín et al. 2013; Rupke \& Veilleux 2013b), and molecular (e.g. Fischer et al. 2010; Feruglio et al. 2010; Sturm et al. 2011; Veilleux et al. 2013; Cicone et al. 2012, 2014; Rupke \& Veilleux 2013a; Spoon et al. 2013). For the ionised and neutral gas, the outflows in ULIRGs with AGN show velocity widths (FWHM) and shifts of up to $1700 \mathrm{~km} \mathrm{~s}^{-1}$ and $2000 \mathrm{~km} \mathrm{~s}^{-1}$, respectively ${ }^{1}$ (Rodríguez Zaurín et al. 2013; Rupke \& Veilleux 2013b, hereafter RZ13 and RV13). These numbers are substantially smaller for ULIRGs with no detected AGN activity (FWHM typically less than $500 \mathrm{~km} \mathrm{~s}^{-1}$, RV13, RZ13). In the molecular phase, the CO emission and/or $\mathrm{OH}$ absorption lines show velocity shifts of up to $1000 \mathrm{~km} \mathrm{~s}^{-1}$ in some ULIRGs with AGN activity (Sturm et al. 2011; Veilleux et al. 2013; Cicone et al. 2014). As in the cases of the ionised and neutral phases, the velocity shifts are smaller for those ULIRGs powered by starbursts (e.g. Veilleux et al. 2013; Cicone et al. 2014).

Recent integral field spectroscopic (IFS) studies of local samples of ULIRGs have been used to investigate not only the kinematics but also the spatial structures and the geometries of these outflows (e.g. Colina et al. 1999; Bedregal et al. 2009; Westmoquette et al. 2012, RV13). For example, RV13 found a variety of outflow morphologies that range from collimated bipolar winds, sometimes in the form of "super-bubbles", to less collimated shocks extending on kiloparsecs scales in the galaxies. However, these studies are usually concentrated in the central few kiloparsecs of the objects, and therefore, it is possible that they do not sample the full extent of the outflowing material. In addition, IFS data are usually limited to a relatively narrow wavelength range, making it hard to determine the ionisation mechanism responsible for the outflows. Due to these limitations, the mass outflow rates and kinetic powers derived from these studies also remain uncertain.

As one of the closest ULIRGs, Mrk273 $\left(z=0.0373, L_{\mathrm{IR}}=\right.$ $\left.10^{12.21} L_{\odot}\right)$ represents a key target for studies aimed at understanding the nature of ULIRGs and their associated outflows. This late-merger system shows an impressive tidal tail extending over $40 \mathrm{kpc}$ to the south of the nuclear region. It has been studied extensively at all wavelengths from the UV to the X-rays (e.g. Condon et al. 1991; Soifer et al. 2000; Armus et al. 2007; Howell et al. 2010; Rodríguez Zaurín et al. 2010; Iwasawa et al. 2011; U et al. 2013). At near- and mid-IR wavelengths, two nuclei become apparent, commonly referred to as northern $(\mathrm{N})$ and south western (SW) components (Majewski et al. 1993; Knapen et al. 1997; Scoville et al. 2000; Soifer et al. 2000). An additional third nuclear structure, referred to as the south eastern (SE) component, emerges at radio wavelengths (Condon et al. 1991). The N nucleus is the strongest radio source (Carilli \& Taylor 2000; Bondi et al. 2005) and makes the main contribution to the MIR emission (Soifer et al. 2000). Radio and CO(2-1) observations suggest that the $\mathrm{N}$ nucleus is the site of the dense molecular gas disk of $\sim 10^{9} M_{\odot}$ containing a compact, powerful starburst formed by several luminous supernovae or supernovae remnants (Condon et al. 1991; Carilli \& Taylor 2000; Bondi et al. 2005). However, based on their recent AO IFS near-IR observations of the sources, $U$ et al. (2013) found evidence for a supermassive black hole of mass $(1.04 \pm 0.1) \times 10^{9}$ solar masses in the $\mathrm{N}$ nucleus. In addition, the detection of enhanced Fe K emission at the same location suggests that this nucleus might contain a heavily obscured AGN (Iwasawa et al. 2011).

\footnotetext{
1 Throughout the paper, when we refer to the outflow velocity shifts from the work of Rupke and collaborators, we specifically mean the $v_{50 \%}$ values as defined in their papers.
}

The SW component coincides with the location of the hard X-ray point source and is identified as the host of the AGN (Iwasawa et al. 2011). On the other hand, although the SE component was first identified as a star cluster based on NICMOS images, the strength of the [SiVI] at this location suggests that this region is at least partially ionised by an AGN that is perhaps located in the $\mathrm{N}$ or SW nucleus (U et al. 2013).

The dominant power source responsible for the IR luminosity of Mrk273 remains uncertain. While the strong [O IV] and [Ne V] emission relative to [Ne II] suggest heating by an AGN as the dominant mechanism (Genzel et al. 1998; Veilleux et al. 2009), the $L(\mathrm{MIR}) / L(\mathrm{FIR})$ ratio is consistent with starburst activity powering almost the entire IR luminosity of the source (Veilleux et al. 2009). In addition, other diagnostics based on the equivalent width of the PAH feature at $7.7 \mu \mathrm{m}$ suggest that the contributions to the heating of the dust by the AGN and starburst components are actually similar (Veilleux et al. 2009).

The nuclear kinematics of the source have been investigated in the past using IFS techniques (Colina et al. 1999, RV13). The RV13 study of the central $4.5 \times 6 \mathrm{kpc}$ revealed the presence of an "extended" outflow plus a bipolar superbubble, along with a rotating component that traces the $\mathrm{CO}(1-0)$ rotation curve of Downes \& Solomon (1998). With projected velocities of up to $-1500 \mathrm{~km} \mathrm{~s}^{-1}$, the superbubble is aligned in the N-S direction and is likely to by ionized by an AGN.

Although much attention has been paid to the nuclear regions of Mrk273, little work has been done on the morphology, kinematics, and ionisation of the gas at larger scales. In this paper we present new imaging and spectroscopic observations of Mrk273 taken with the Hubble Space Telescope (HST) and the Isaac Newton Telescope (INT). We use these combined datasets to relate the properties of the ionised gas emission in the nuclear regions to those on more extended scales. In this way, we aim to establish whether the warm, AGN-driven outflows are important for the overall evolution of the host galaxy. Throughout the paper we adopt $H_{0}=68 \mathrm{~km} \mathrm{~s}^{-1}, \Omega_{\mathrm{m}}=0.29$ and $\Omega_{\Lambda}=0.71$. At the redshift of the source, this gives a luminosity distance of $D_{\mathrm{L}}=171.5 \mathrm{Mpc}$ and a scale of $0.762 \mathrm{kpc} \operatorname{arcsec}^{-1}$.

\section{Observations and data reduction}

\subsection{Imaging data}

\subsubsection{HST data}

New HST/ACS images of Mrk273 were taken during Cycle 20 as a part of a larger program aimed at studying the AGNinduced outflows in a sample of local ULIRGs (GO:12934, PI: C. N. Tadhunter). The Wide Field Channel (WFC, 0.049 arcsec pixel $^{-1}$ ) on the ACS was used in combination with the FR505N narrow-band ramp filter and the F550M mediumband filter. The former image was centered on the redshifted [OIII] $\lambda 5007$ (hereafter [OIII]) emission line, while the F550M medium-band filter was centered on the nearby continuum towards redder wavelengths. Details of the imaging observations are shown in Table 1.

The data were reduced using the standard data reduction pipeline procedures which employ two packages: the CALACS package, which includes dark subtraction, bias subtraction, and flat-field correction and produces calibrated images, and the MULTIDRIZZLE package, which corrects for distortion and performs cosmic ray rejection. Any remaining cosmic rays were removed manually using the routines IMEDIT in IRAF and/or CLEAN within the STARLINK package FIGARO. 
Table 1. Log of the imaging observations of Mrk273 $(z=0.0373)$ used for this paper.

\begin{tabular}{llllll}
\hline \hline Telescope & Camera & Filter & $\begin{array}{l}\lambda_{\mathrm{c}} \\
(\AA)\end{array}$ & $\begin{array}{l}\Delta \lambda \\
(\AA)\end{array}$ & $\begin{array}{l}\text { Exp time } \\
(\mathrm{s})\end{array}$ \\
\hline HST & ACS/WFC & $F 550 M$ & 5581.5 & 384 & 716 \\
& & $F R 505 N$ & 5175.6 & 92 & 1356 \\
INT & WFC & $\# 228$ & 6813 & 93 & 2800 \\
\hline
\end{tabular}

To convert into physical units, we used the PHOTFLAM header keyword. PHOTFLAM is the sensitivity conversion factor and is defined as the mean flux density $F_{\lambda}$ (in units of erg cm ${ }^{-2} \AA^{-1}$ counts $^{-1}$ ) that produces 1 count per second for a given HST observing mode. Since drizzled ACS images are in units of counts $\mathrm{s}^{-1}$, these may simply be multiplied by the PHOTFLAM value to obtain the flux in units of $\operatorname{erg~cm}{ }^{-2} \mathrm{~s}^{-1} \AA^{-1}$. The error associated with the flux calibration, including both the photon noise and calibration uncertainty, is $\sim 5 \%$ for both the continuum and the [OIII] image.

At this stage, it is important to add a caveat about the use of the PHOTFLAM parameter. While PHOTFLAM is adequate when the flux is approximately constant through the bandpass, it might not be adequate when the spectrum of the source shows strong emission lines within the bandwidth, which is the case of our [OIII] image. Therefore, to check the accuracy of the calibration of the HST images, we compared them with the Rodríguez Zaurín et al. (2009) WHT/ISIS long slit spectroscopic observations of the source.

With this aim, it is crucial to know the precise location of the slit during the observations. We first convolved the HST images with a Gaussian profile to simulate the seeing conditions during the spectroscopic observations. Spatial profiles of width identical to the slit-width of the spectra $(1.5 \mathrm{arcsec})$ were then extracted from the images in steps of 2 pixels $(0.1 \mathrm{arcsec})$. These spatial profiles were compared with those of spatial slices extracted from the spectra using wavelength ranges selected to match those of the HST filters until a match was found. Following this procedure, we were able to find the location of the slits on the image with a precision of 5 HST pixels $(0.25 \mathrm{arcsec})$. Once the location of the slit was known, we integrated the emission over the spatial extent of the galaxy covered by the slit for both the HST and the WHT spatial profiles described before.

The results obtained from this procedure are two flux measurements that can be directly compared to check the consistency between the flux calibrations. If we refer to these flux values as $F_{\mathrm{HST}}$ and $F_{\mathrm{WHT}}$, we find that $F_{\mathrm{HST}} / F_{\mathrm{WHT}}$ is equal to 1.05 and 1.10 for the continuum and [OIII] observations respectively. Given the uncertainties associated with the process (e.g. the varying seeing during the spectroscopic observations, or the 5 pixel uncertainty in the slit position) we find that the flux calibrations for the HST and the WHT observations are in excellent agreement.

Once the images were calibrated in flux, we subtracted the continuum from the [OIII] image to end up with an image that traces "pure" [OIII] emission. Prior to this task, it was necessary to align the images. To that aim, we used a series of IRAF routines. In the first place, we used the task SREGISTER, which registers an image to a reference image using celestial coordinate information in the headers. In addition, there are three foreground stars in the ACS/WFC field of view used for the Mrk273 observations. Therefore, it was possible to use the tasks GEOMAP and GEOTRAN to geometrically align the images and refine the final result. The accuracy of the alignment was measured using the routine CENTER that calculates the centroids of the stars in the aligned images. We found that the images are aligned with an accuracy better than 0.02 pixels. The continuum, continuum subtracted, and [OIII]+continuum images are shown in Fig. 1.

\subsubsection{INT/WFC data}

A deep image of Mrk273 was taken in April 2013 using the Wide Field Camera (WFC) mounted on the $2.5 \mathrm{~m}$ INT telescope at the Observatorio del Roque de los Muchachos (ORM), La Palma. The WFC is an optical mosaic camera consisting of four CCDs of $2098 \times 4100$ pixels (0.33 arcsec/pixel). Mrk273 was always observed with CCD4, which has the highest efficiency at red wavelengths. For these observations we used the Filter \#228 $\left(\lambda_{\mathrm{c}}=6813 \AA, F W H M=93 \AA\right)$, which is centered on the $\mathrm{H} \alpha$ emission line ${ }^{2}$ and includes the [NII] $\lambda \lambda 6549,6583$ doublet. To remove the cosmic rays, hot pixels, and other artifacts we took four $700 \mathrm{~s}$ exposures dithered on four positions in the sky. Unfortunately, due to some technical difficulties during the night of the observations, we could not acquire a line-free continuum image in a wavelength range adjacent to $\mathrm{H} \alpha$. Therefore, no continuum subtraction could be performed in the case of the INT/WFC image of Mrk273 presented in this paper. The seeing during the observations, as measured with foreground stars in the images, was $F W H M=1.5-1.6$ arcsec. Details of the INT/WFC observations are shown in Table 1.

We used a series of IRAF routines to perform the standard reduction process, including bias subtraction, flat-field correction, and cosmic ray rejection. Any remaining cosmic rays after the initial reduction were cleaned "manually" using the routines IMEDIT in IRAF and/or CLEAN within the STARLINK package FIGARO. To transform into physical units, we used two standard stars observed with the exact same set-up used for the observations of the galaxy and calculated a factor to transform counts s $\mathrm{s}^{-1}$ into ergs s $\mathrm{s}^{-1} \mathrm{~cm}^{-2}$. The calibration factor obtained for each of the stars is identical and the estimated flux calibration uncertainty is $7 \%$. The flux calibrated INT image is shown in Fig. 2.

Finally, we have followed the same procedure described in the previous section for the HST images and compared the flux calibration of the INT/WFC image with that of the existing WHT/ISIS spectroscopic observations of the galaxy (Rodríguez Zaurín et al. 2009). If we define $F_{\mathrm{WFC}}$ as the integrated flux over the spatial extent of the galaxy covered by the slit in the WFC image, we find that $F_{\mathrm{WFC}} / F_{\mathrm{WHT}}=0.8$, i.e. the flux calibrations for the two observations are in good agreement.

\subsection{Spectroscopic data}

Long-slit optical spectroscopic observations were taken for Mrk273 in May 2013 using the Intermediate Dispersion Spectrograph (IDS) on the INT. The observations were obtained using the R400V grating centered at $5250 \AA$ with the RED +2 CCD. The instrumental set-up resulted in a spatial scale of 0.44 arcsec pix ${ }^{-1}$ and a dispersion of $1.5 \AA \mathrm{pix}^{-1}$. The useful wavelength range is $\sim 4000-7200 \AA$. The data were reduced (bias subtracted, flat field corrected, cleaned of cosmic rays, wavelength calibrated, and flux calibrated) and straightened before extraction of the individual spectra using the standard packages in IRAF and the STARLINK packages FIGARO and

2 We assume a systemic redshift of $z=0.0373$ for the system (see RV13). All the radial velocity shifts presented in this paper are measured relative to this redshift. 

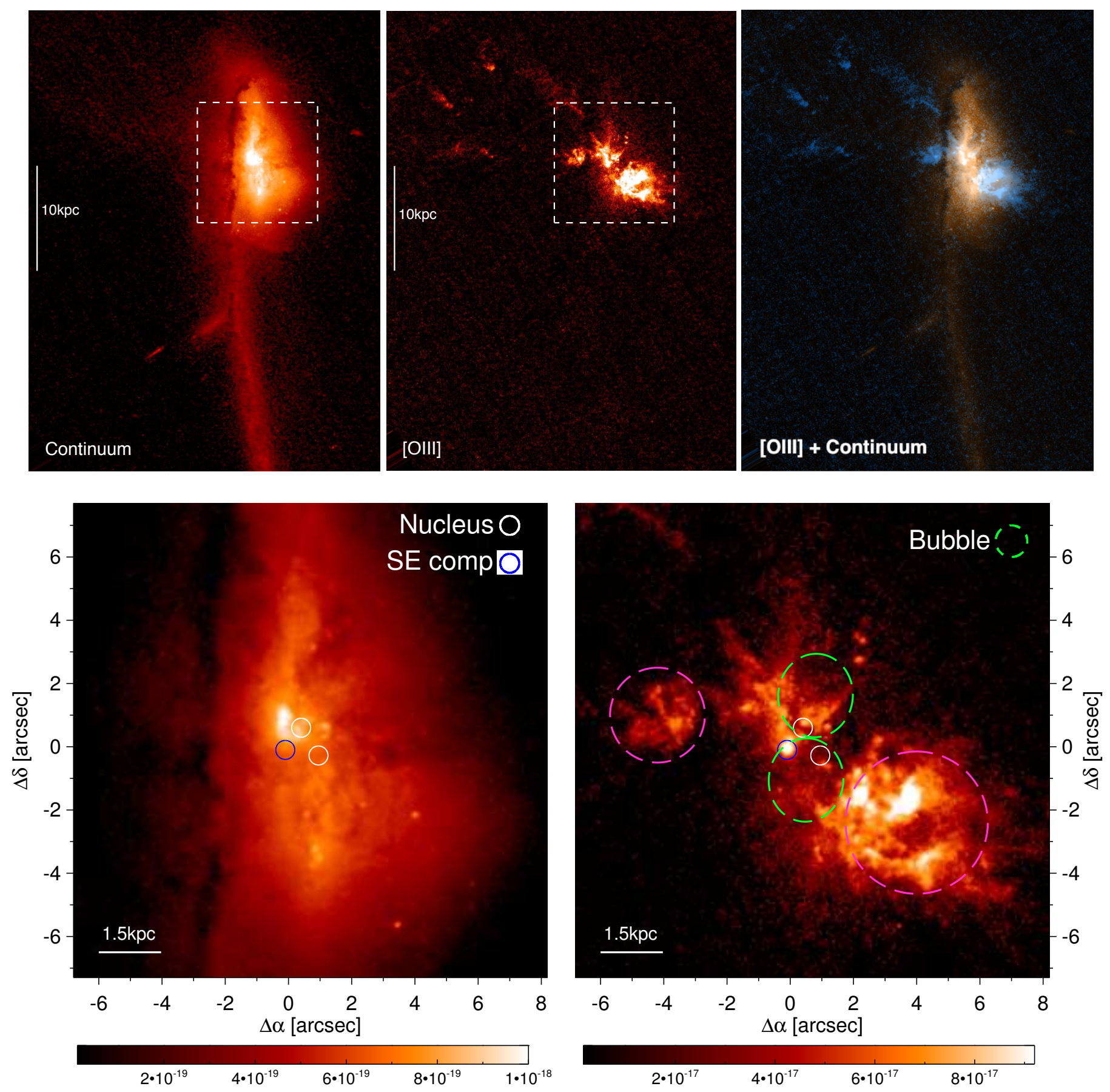

Fig. 1. Upper panel, left to right: HST/ACS continuum, continuum subtracted [OIII], and [OIII] (blue)+continuum (red) images of the galaxy. North is up, east is left. The dashed-line boxes indicated the zoomed region in the lower panel. Lower panel: zoom into the central $15 \times 15$ arcsec region $(11.6 \times 11.6 \mathrm{kpc}$ ) for the continuum (upper-left) and the continuum-subtracted [OIII] (upper-middle) images of the galaxy. The color bars at the bottom of the images indicate the flux in units of erg s $\mathrm{s} \mathrm{cm}^{-2} \AA^{-1}$ and $\mathrm{erg} \mathrm{s}^{-1} \mathrm{~cm}^{-2}$ for the continuum and the [OIII] images respectively. The white circles indicate the locations of the $\mathrm{N}$ and SW nuclear components observed at IR wavelengths, while the blue circle corresponds to the location of the SE component that emerges at radio wavelengths. The green-dashed line ellipses in the continuum subtracted [OIII] image indicate the location of the nuclear superbubble reported by RV13 in their IFS study of the source, while magenta-dashed line circles indicate the location of the so-called Outflow-East and West regions (see text for details). (A color version of this figure is available in the online journal.)

DIPSO. The wavelength calibration accuracy, as determined using the mean shift between the measured and published (Osterbrock et al. 1996) wavelengths of night-sky emission lines, is $\sim 0.35 \AA\left(20 \mathrm{~km} \mathrm{~s}^{-1}\right)$. The spectral resolution, calculated using the widths of the night-sky emission lines (FWHM), is $3.1 \pm$ $0.1 \AA$ at $6300 \AA\left(145 \pm 5 \mathrm{~km} \mathrm{~s}^{-1}\right)$. The estimated uncertainty for the relative flux calibration is $\pm 5 \%$, based on a comparison of the response curves of various spectrophotometric standard stars observed during the observing runs. Finally, the seeing during the observations, measured using stars in the acquisition image, was 0.9-1.2 arcsec. Details of the INT/IDS observations for Mrk273 can be found in Table 2 . 


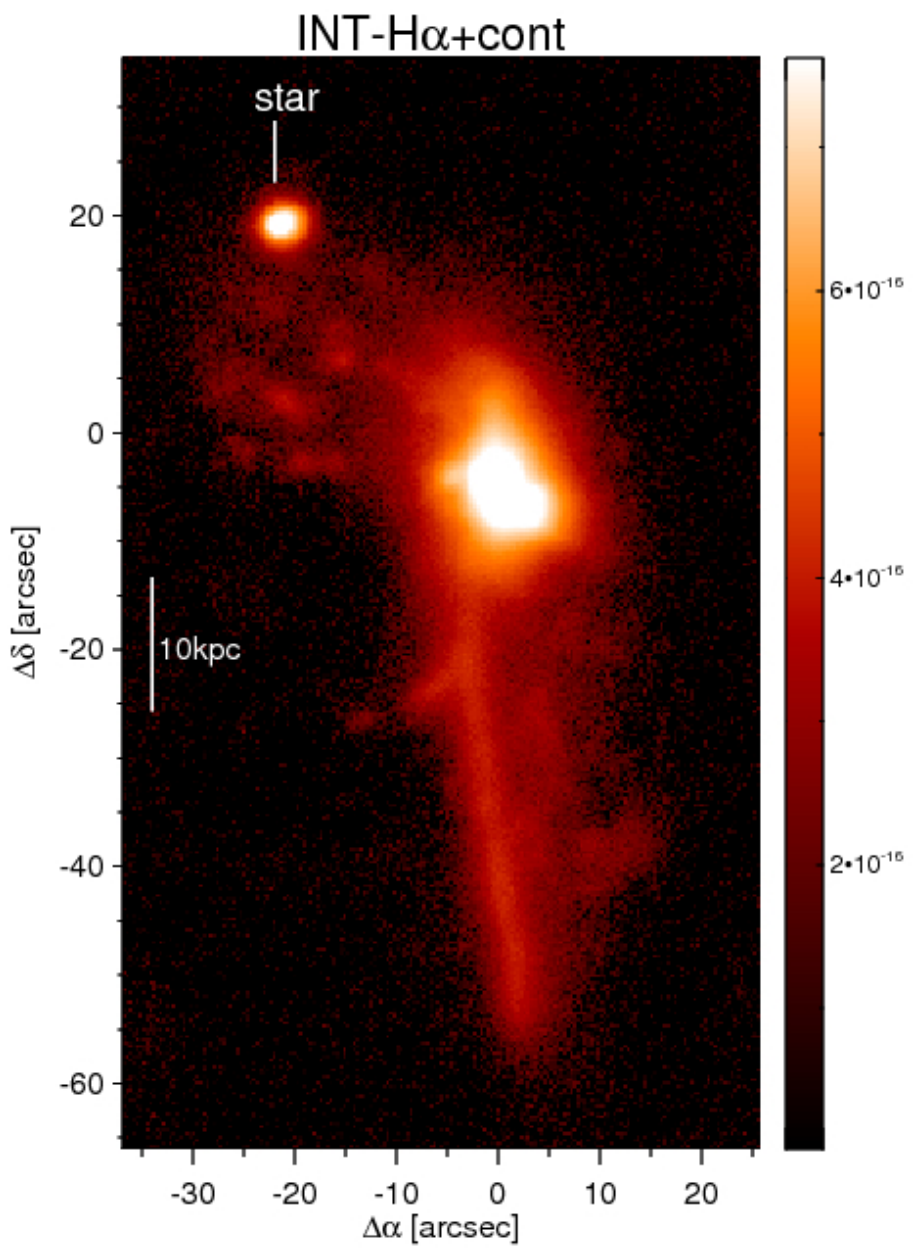

Fig. 2. INT/WFC H $\alpha+[\mathrm{NII}]$ image of Mrk273. No continuum emission was subtracted for this image. The color bar to the right of the image indicates the flux in units of ergs $\mathrm{s}^{-1} \mathrm{~cm}^{-2}$. The bright point source to the northeast of the galaxy is a star. (A color version of this figure is available in the online journal.)

Table 2. Log of the INT/IDS spectroscopic observations of Mrk273 $(z=0.0373)$ used for this paper.

\begin{tabular}{lllll}
\hline \hline Slit Position & $\begin{array}{l}\text { Slit PA } \\
\text { degrees }\end{array}$ & $\begin{array}{l}\text { Exp time } \\
\text { (s) }\end{array}$ & Airmass & $\begin{array}{l}\text { Seeing } \\
\text { (arcsec) }\end{array}$ \\
\hline Pos1 & 70 & 6000 & 1.24 & $0.9-1.1$ \\
Pos2 & 56 & 7200 & 1.14 & $0.9-1.2$ \\
Pos3 $^{\star}$ & 70 & 7200 & 1.24 & $0.9-1.2$ \\
Pos4 $^{\star}$ & 56 & 6000 & 1.14 & $0.9-1.1$ \\
\hline
\end{tabular}

Notes. The seeing (FWHM) was estimated using field stars in the acquisition images. ${ }^{\star} \star$ These two slits (Pos3 and Pos4) were placed at exactly the same PA as Pos 1 and Pos2 respectively, but were shifted by 1 arcsec to the north. For clarity, the slit positions 3 and 4 are not shown in Fig. 3.

For these observations, we used four slit positions selected to include the main ionised gas features observed in our ACS-[OIII] image of the galaxy. Two slits were placed at PA 70 and another two at PA 56. The relative shift between each pair of slits at the same PAs was 1 arcsec to the north. The slits cover the bright [OIII] emission in the nuclear region of the galaxy, as well as a significant fraction of the faint, very extended [OIII] emission observed towards the east of the nuclear region.
The extraction apertures for each slit position were selected based on spatial cuts of the 2D-frames in wavelength ranges chosen to include the $[\mathrm{OIII}] \lambda 5007$ and the $\mathrm{H} \alpha+[\mathrm{NII}]$ emission lines. The slit position and the corresponding extraction apertures for Pos 1 and 2 are shown in Fig. 3, where they are overplotted onto the continuum subtracted [OIII] image of the galaxy. To simulate the seeing conditions during the spectroscopic observations, the [OIII] image in the figure has been convolved with a Gaussian of $F W H M=1$ arcsec.

Since we aim to compare our results with those of previous studies of the galaxy, it is crucial to know the precise location of the slits. With this aim, we followed again the same procedure as described in Sect. 2.1.1 to find the location of the slits on the HST images with a precision of 5 pixels $(0.25 \mathrm{acsec})$. In addition, we checked the consistency of the flux calibrations for the entire dataset used in this paper by comparing the INT/IDS observations with the HST images. If we define $F_{\text {IDS }}$ as the integrated flux over a espatial slice extracted from the spectra using wavelength ranges selected to match those of the HST filters, we find that $F_{\mathrm{HST}} / F_{\text {IDS }}$ is equal to 0.87 and 0.78 for the continuum and [OIII] observations respectively. Overall, the flux calibrations for the different datasets used for this paper show a high degree of consistency.

\section{Results}

\subsection{Imaging}

\subsubsection{HST imaging: the detailed morphology of the ionised gas emission}

The most remarkable feature visible in our emission-line-free, F550M continuum image of the galaxy (upper-left panel in Fig. 1) is the well-known extended tail to the south of the galaxy. Note that the $F 550 M$ continuum image of the galaxy does not cover the full extent of the tail ( $\sim 36 \mathrm{kpc}$ or $\sim 46^{\prime \prime}$. See for example, Kim et al. 2002, their Fig. 1). The image also shows some weak, but significant continuum emission towards the northeast of the nuclear region and to the west of the tidal tail. The lowerleft panel in our Fig. 1 shows the morphology of the continuum emission in the central $15 \times 15$ arcsec. There is a large number of bright knots, clumps and other irregular condensations around the brightest "L-shaped" structure close to the N and SE nuclear components. All of these structures are crossed by dust features, with the most prominent dust lane extending in the north to south direction to the east of the nuclear region, and then along the tidal tail.

Figure 1 shows the continuum subtracted [OIII] image of the galaxy. This is strikingly different from the continuum image. The first notable structure in the image is the spectacular system of extended filaments and clumps to the east of the galaxy. Such clumps and filaments extend $\sim 25^{\prime \prime}(\sim 19 \mathrm{kpc})$ in our [OIII] image; the average position angle of the structure, as measured relative to the $\mathrm{N}$ nucleus, is $\sim 68^{\circ}$, and the range of position angles covered by the structure is $\sim 50^{\circ}$.

To better study the morphology of the [OIII] emission in the nuclear region, the lower-right panel in Fig. 1 concentrates on the [OIII] emission from the central $15 \times 15$ arcsec of the galaxy. The locations of the N, SW and SE components are indicated in the figure with open circles. It is notable that the [OIII] emission is not particularly enhanced at the location of the $\mathrm{N}$ and SW nuclei, which is likely related to reddening effects. However, coinciding with the location of the SE component we find the brightest and most compact condensation of 

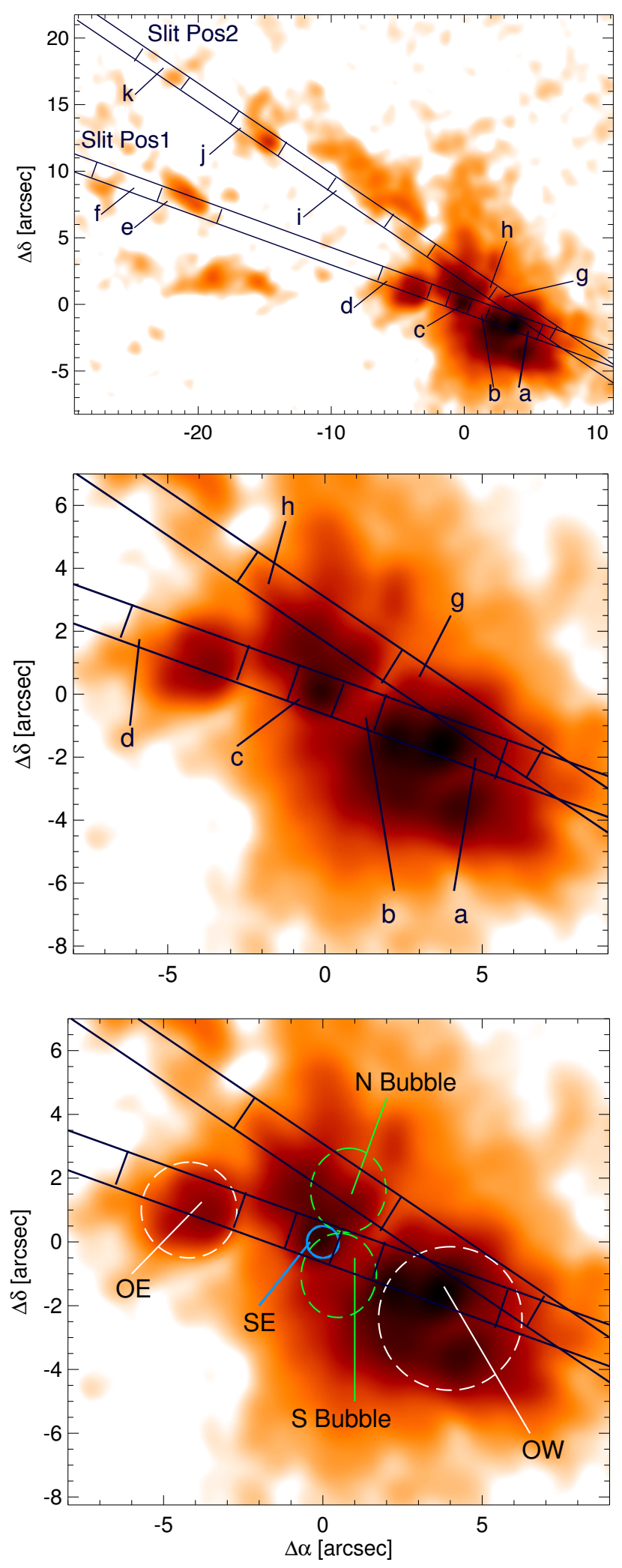

Fig. 3. Upper panel: HST continuum subtracted [OIII] image of the galaxy showing the location of the slits and the extraction apertures. The images have been convolved with a Gaussian of $F W H M=1^{\prime \prime}$ to simulate the seeing conditions during the spectroscopic observations. Middle and lower panels: these two figures are a zoom into the nuclear region of the galaxy. The figures show in detail the main regions of interest that are later analyzed in the paper (see the text for details) and the apertures that cover those regions. (A color version of this figure is available in the online journal).
[OIII] emission observed in our images. To estimate the size of this feature, we fitted a 2D Gaussian to the region. The resulting FWHM $\left(F W H M_{\text {unc }}\right)$ was corrected for instrumental width ( $\left.I_{\text {FWHM }}\right)$ by subtracting the two values in quadrature ${ }^{3}$. The corrected FWHM is $F W H M_{\text {corr }}=0.13 \pm 0.02 \operatorname{arcsec}(99 \pm 15 \mathrm{pc})$. Therefore, although the SE component is compact, it is resolved in our observations.

The lower-right panel in Fig. 1 also shows a schematic of the nuclear-superbubble model proposed by RV13 in their recent IFS study of the galaxy. Interestingly, the figure shows a "U-shaped" structure of diffuse [OIII] emission that closely follows the structure of the northern, near side of the bipolar superbubble (referred here as "N-Bubble"). However, the southern, far side of the bubble ("S-Bubble") is not clearly visible in our image, which is likely related to reddening effects. Finally, on larger scales, but still within the central $\sim 15$ arcsec, there are two high surface brightness structures of enhanced [OIII] emission extended $\sim 5 \operatorname{arcsec}(4 \mathrm{kpc})$ to the east and west of the nuclear region, almost perpendicular $\left(\mathrm{PA} \sim 80^{\circ}\right.$ ) to the RV13 nuclear supperbubble. Overall, the structure of the [OIII] emission in the nuclear regions is highly complex. A detailed spectroscopic study of the different structures described in this section is carried out later in the paper.

\subsubsection{INT imaging: low surface brightness emission at large scales}

The HST images of Mrk273 show no evidence for [OIII] emission at or around the tidal tail to the south of the galaxy. There are two possible explanations for this finding. The most straightforward explanation is that there is no ionised gas at these locations. This would be consistent with the results of Rodríguez Zaurín et al. (2009) from their detailed study of the stellar populations in the galaxy. These authors found that the optical (3000-7500 $\mathrm{A}$ ) continuum of the source along the tidal tail is remarkably uniform, and is adequately modelled using a stellar population of age $\sim 700$ Myr that dominates the optical emission, with a small contribution from a young (i.e. ionising) stellar population.

However, given the size of the resolution element of the ACS-WFC, it is possible that some low-surface-brightness emission line features are not detected in our HST images. To detect such faint features with the ACS camera, one would require substantially longer exposure times than those used for our observations. An alternative is to use deep, lower resolution images. In this context, Fig. 2 shows our deep, $\mathrm{H} \alpha+[\mathrm{NII}]$ image of the galaxy taken with the Wide Field Camera (WFC) at the INT telescope. The seeing during these observations was $\sim 1.6^{\prime \prime}$. Therefore, none of the detailed nuclear structure visible in the HST images is distinguishable in Fig. 2. However, the figure shows diffuse emission extending $\sim 23 \mathrm{kpc}$ to the east of the nuclear regions and $\sim 10 \mathrm{kpc}$ to the west of the southern tidal tail.

Unfortunately, we do not have a deep INT/WFC line-free continuum images to allow accurate quantification of the continuum contribution to the extended structures visible in the INT narrow-band image. However, we note that the filamentary structure to the west of the southern tidal tail has a peak surface brightness that is $\sim 50 \%$ of that of the tidal tail itself. If this were

\footnotetext{
3 The instrumental width $\left(I_{\mathrm{FWHM}}\right)$ was measured using the same $2 \mathrm{D}$ Gaussian fit for two stars in the field. We obtained $I_{\mathrm{FWHM}}=0.115 \pm$ 0.005 arcsec. The FWHM of the SE component before the correction is $F W H M_{\text {unc }}=0.174 \pm 0.005$ arcsec. The corrected FWHM is calculated as $F W H M_{\text {corr }}=\sqrt{F W H M_{\text {unc }}^{2}-I_{\mathrm{FWHM}}^{2}}$.
} 
predominantly a continuum structure, we would expect it to be visible at a similar contrast level in our F550M continuum image and HST broad-band images of the source that are continuumdominated (e.g. the HST F435W and F814W images presented in Mazzarella \& Boroson 1993; Hibbard \& Yun 1996; Kim et al. 2002). Because the diffuse emission to the west of the southern tidal tail is much fainter in the latter images, this provides strong evidence that the western filaments that run parallel to the main tidal tail are predominantly emission line structures.

We further note that the western filamentary structure partially follows the morphology of the extended X-ray nebula reported by Iwasawa et al. (2011). Their results, which are based on new Chandra X-ray observations of the source, are consistent with the tidal tail being located in front of the soft X-ray nebula in our line of sight, such that the soft X-ray emission is partially absorbed by the cold gas in the tidal tail. Moreover, Iwasawa et al. (2011) derived a lower limit on the column density of $N_{\mathrm{H}} \sim 10^{22} \mathrm{~cm}^{-2}$, which is similar to that of edge-on galaxy disks and consistent with other measurements of disk shadowing (Barber et al. 1996). Iwasawa et al. (2011) concluded that the source of the soft X-ray emitting gas is likely to be star formation in the edge-on disk, which would be, a priori, consistent with our INT/WFC image. In this scenario, the highly reddened, young stellar populations in the disk would not make a large contribution to the visible optical emission at these locations, which would explain the Rodríguez Zaurín et al. (2009) results for the stellar populations along the tail. Note, however, that although $\mathrm{H} \alpha+[\mathrm{NII}]$ emission is only detected to the west of the tail in our INT/WFC image, Iwasawa et al. (2011) found soft X-ray emission on both sides of the tidal tail. Future, deeper $\mathrm{H} \alpha$ images of the galaxy will help to investigate in greater detail the extended ionised gas emission around the tail and its relation with the soft $\mathrm{X}$-ray emission at these locations.

\subsection{Spectroscopy}

\subsubsection{Continuum emission subtraction}

Prior to the modelling of the emission line profiles in the nuclear regions, the spectra were shifted to the galaxy rest frame $(z=0.0373:$ RV13). Since we aim to study both the kinematics and the ionisation mechanisms in Mrk273, it is important to subtract the underlying stellar continuum prior to modelling the emission line profiles. This might be an important issue for the $\mathrm{H} \beta$ and $\mathrm{H} \alpha$ emission lines, which are are the most affected by the stellar absorption. Unfortunately, our INT/IDS spectra do not have sufficient wavelength range coverage to carry out a detailed analysis of the stellar populations. For this reason, we used the results of spectral synthesis model fits to the wider-coverage (3000-7500 $\AA$ ) WHT/ISIS spectra of this source, as presented in Rodríguez Zaurín et al. (2009), to guide our fits to the stellar continuum of the INT/IDS data.

Rodríguez Zaurín et al. (2009) found that the stellar populations across the full extension of the galaxy covered by their slit (see Fig. 3) were remarkably uniform in terms of their ages, with no evidence for a significant age gradient. The best-fitting models in the central regions (their apertures D, E and F) comprise a $\sim 0.7-1.0$ Gyr $(E(B-V) \leq 0.4)$ stellar population and a significant contribution from a reddened $\$ 50 \mathrm{Myr}(E(B-V) \leq$ 1.0) stellar population (see Rodríguez Zaurín et al. 2009, for details). Therefore, we took the approach of assuming a similar mix of stellar populations to correct for the stellar continuum the INT/IDS spectra. This approach was used only for those apertures that sample regions with significant continuum emission, as observed in the ACS continuum image of the source. These are: AP-a, b, c, d, g, and h.

The stellar population within the apertures with significant stellar continuum were modelled using a similar technique to that described in Rodríguez Zaurín et al. (2009), but using a smaller number of discrete values for the age and reddening of the stellar populations (see Rodríguez Zaurín et al. 2009 for details). Although it is beyond the scope of this paper to perform a detailed analysis of the stellar populations in the galaxy, we emphasise that models including a $0.7 \mathrm{Gyr}(E(B-V)=0.2)$ and a $<10 \mathrm{Myr}(0.5<E(B-V) \leq 1.0)$ stellar population of varying contribution (25-90\%) accurately reproduce the stellar continumm emission from the galaxy for these six apertures.

Overall, we find that subtracting the stellar continuum is important in better constraining the properties of the different kinematic components, especially for the $\mathrm{H} \beta$ emission line. For example, three kinematic components are required to adequately model the $\mathrm{H} \alpha$ or the [OIII] $\lambda \lambda 4959,5007$ emission lines at almost all locations in the nuclear regions. However, before subtracting the stellar continuum, there are cases where only two of these components are required when modelling $\mathrm{H} \beta$. In these cases, we often find that the third kinematic component, typically the one that makes the smallest contribution to the overall $\mathrm{H} \alpha$ emission, clearly emerges once the stellar emission has been subtracted from the spectra. The change in flux for the kinematic components detected before subtracting the stellar continuum is small for $\mathrm{H} \beta$, a factor of $\lesssim 1.3$. In the case of the $\mathrm{H} \alpha$ emission line, the effects of correcting from stellar absorption are negligible.

\subsubsection{Fitting the emission line profiles}

Following continuum subtraction, we used a combination of DIPS $O$ and the IDL MPFIT (Markwardt 2009) code to fit Gaussian profiles to the emission lines. Our modelling approach is described in detail in Rodríguez Zaurín et al. (2013). To summarise, we first selected a prominent emission line that is sufficiently bright and unblended to allow the generation of a model that could be used to fit all the other emission lines. In this context the strong [OIII] $\lambda \lambda 4959,5007$ lines usually have high $\mathrm{S} / \mathrm{N}$ in our spectra, are in a region clean from any atmospheric absorption, and are not blended with other emission lines. We fit these emission lines with the minimum number of Gaussian components required to produce an acceptable fit. The number of Gaussian components required in the model for each line was determined using both an assessment of the reduced Chi squared $\left(\chi_{\text {red }}^{2}\right)$ values and visual inspection of fits overlaid on the data. For example, models comprising two kinematic components resulted in $\chi^{2}$ red values of 2.4-4 for Ap-a, b, d, and h (see Figs. 3 and 4). Including a third, more kinematically disturbed, component resulted in a significant decrease in the $\chi^{2}$ red, down to values of one or less. A similar reduction in $\chi^{2}$ red was not seen when we added a second or third kinematic component in the cases of the SE compact structure (Ap-c) and in regions covering the extended arc structures to the east (e.g. Ap-j).

In addition, when fitting the [OIII] lines, we used three constraints in accordance with atomic physics: i) the flux ratio between [OIII] $\lambda 5007$ and [OIII] $\lambda 4959$ was set at 2.99:1 (based on the transition probabilities); ii) the widths of the corresponding kinematic components of each line were forced to be equal; iii) the shifts between the corresponding components of each line were fixed to be $48.0 \AA$. We refer to the model fitted to the [OIII] lines in this way as the OIII] model in what follows. 

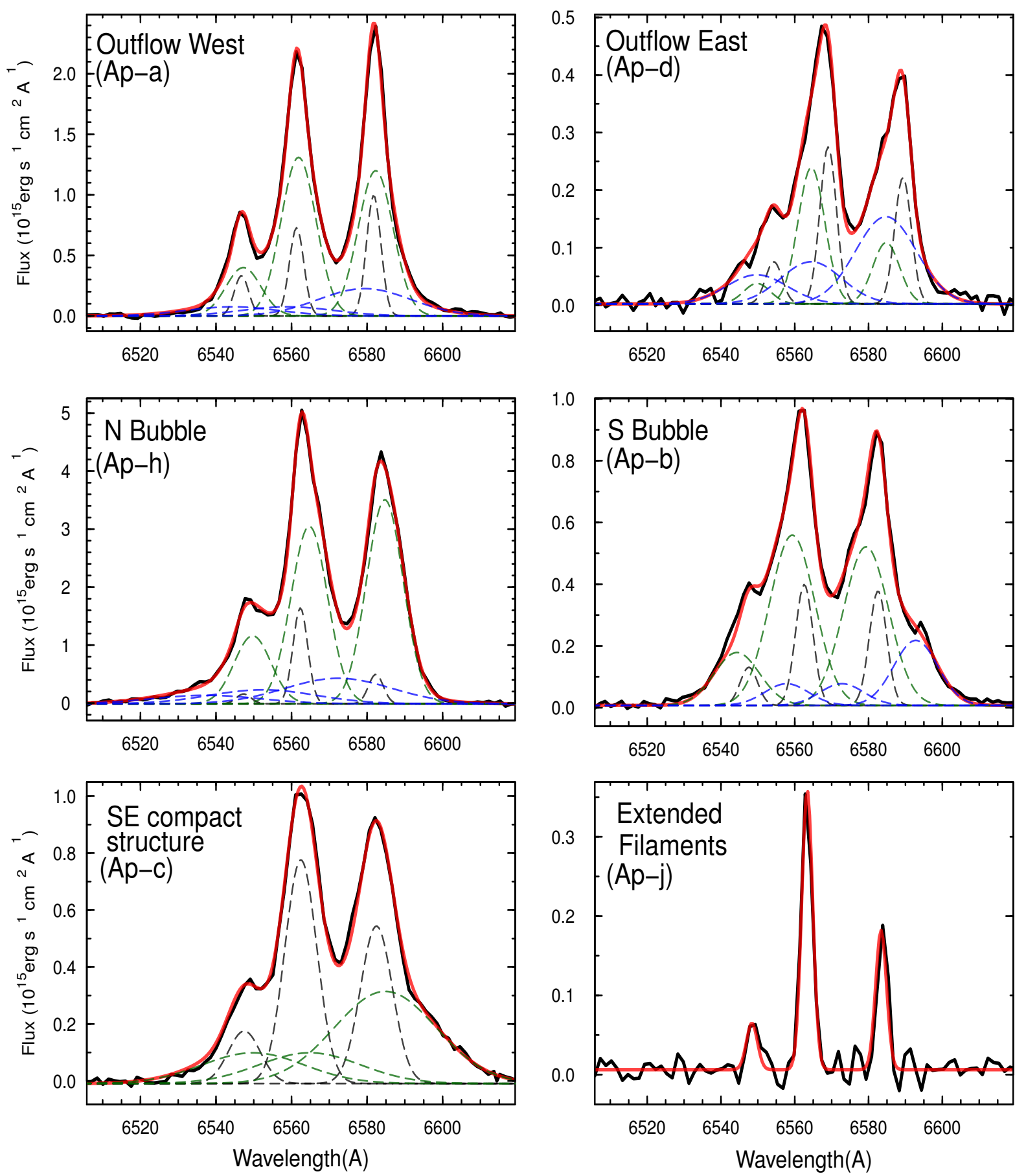

Fig. 4. Emission line modelling results for the $\mathrm{H} \alpha\left(\lambda_{\text {rest }}=6563 \AA\right)$ and the $[\mathrm{NII}]$ emission lines $\left(\lambda_{\text {rest }}=6548\right.$ and $6583 \AA$ ). The black solid line represents the data, while the red solid line represents the overall fit to the emission lines. Black, green and blue dashed lines correspond to the different kinematic components in order of increasing FWHM. The region of the galaxy corresponding to each of the emission line profiles, and the extraction aperture that samples that region, are also indicated in the figure. (A color version of this figure is available in the online journal.)

Once the [OIII] model has been derived, we attempted to model the other prominent emission lines in the spectra with the same kinematic model (velocity widths and shifts) as [OIII], leaving the relative fluxes in the kinematic sub-components to vary. As well as the constraints derived from the [OIII] model, we further constrained the fits to other doublets in accordance with atomic physics (see RZ13 for details). After a series of iterations, the initial [OIII] model was refined, so that the final kinematic model is the best-fitting model that adequately reproduces all the prominent emission lines in the spectra. The velocity widths derived from the fits were quadratically corrected for the instrumental profile, and all linewidths and radial velocity shifts were corrected to the rest frame of the object $(z=0.0373)$.

Table 3 shows the velocity shifts, widths, line fluxes and emission line ratios, for slit positions Pos1 and Pos2. We note that the line ratios in the table have not been corrected for reddening. This is because estimating the reddening is challenging based just on our existing data. The $\mathrm{H} \alpha$ is in a blend, and there are potentially degeneracies involved in the fits that may affect the accuracy of the line ratio measurements, particularly in the 


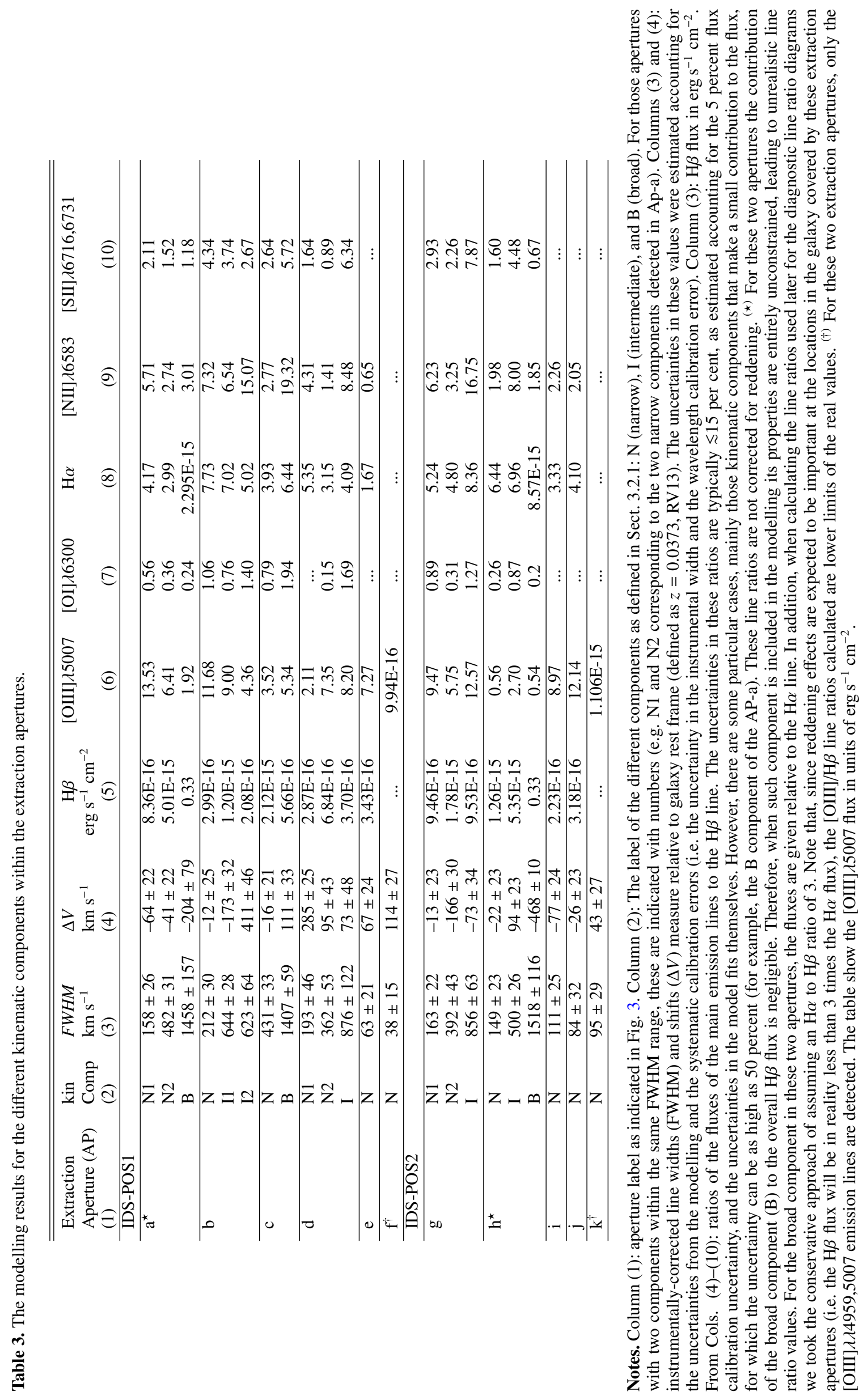


case of the highly complex emission line kinematics present in the near-nuclear regions of Mrk273.

As mentioned in Sect. 2.2, two additional long-slit spectra were taken at the exact same PAs as Pos 1 and Pos2 but shifted one arcsec to the north. These are referred as Pos 3 and Pos4 in Table 2. For each of these two additional slit positions we extracted a set of four extraction apertures, respectively, that sample similar regions to those sampled by Pos 1 and Pos 2 (but 1 arcsec immediately to the north). The modelling results are generally consistent with those for Pos 1 and Pos 2 and, therefore, are not shown in Table 3.

To compare with the recent RV13 optical IFS study, which involves fits to the $\mathrm{H} \alpha+[\mathrm{NII}]$ complex, Fig. 4 shows the fits to the $\mathrm{H} \alpha+[\mathrm{NII}]$ emission lines profile for six extraction apertures selected to sample the main regions of interest, as observed in our HST images. These are Ap-a, Ap-b, Ap-c, Ap-d, Ap-h and Ap-j (see Fig. 3 for their location in the galaxy). In Fig. 4, the apertures are also labelled according to the region that they cover. Ap-a and Ap-d sample the outflowing gas to the west and east of the nuclear region, and therefore are labelled as OutflowWest and Outflow-East respectively. Ap-b and Ap-h sample the lobes of the nuclear superbubble found by RV13 to the south and north of the nuclear region and are labelled as N-Bubble and S-Bubble respectively. Finally, Ap-c and Ap-j sample the compact $S E$ structure in the nuclear region and the clumpy filaments of extended emission. The latter are labelled as the SE compact structure and extended filaments, respectively. See Figs. 3 and 4 for details.

For ease of reference in the following sections, we use the following scheme to label kinematic components, based on line widths (FWHM):

- narrow: $F W H M<500 \mathrm{~km} \mathrm{~s}^{-1}$;

- intermediate: $500 \leq F W H M<1000 \mathrm{~km} \mathrm{~s}^{-1}$;

- broad: $F W H M \geq 1000 \mathrm{~km} \mathrm{~s}^{-1}$.

\section{Discussion}

\section{1. lonised gas kinematics}

Although the spatial resolution of the long-slit observations is not as good as that of the HST images, they are useful in gaining a general idea of the ionised gas kinematics at different locations in the galaxy. In this context, Fig. 5 shows the velocity shifts $(\Delta V)$ and widths (FWHM) along the slits Pos1 and Pos2. For this figure, we have used extraction apertures of two pixels (i.e., 0.88 arcsec) except in the very extended regions, where we used the apertures Ap-e and Ap-f for Pos1 and Ap$\mathrm{i}, \mathrm{Ap}-\mathrm{j}$, and Ap-k for Pos2. Since the kinematics of the galaxy are highly disturbed across the slits, the selection of a reference point $(D=0$ arcsec $)$ is rather arbitrary. In the case of Pos1, the reference point is the centroid of the SE structure (i.e. RA $=0$ and Dec $=0$ in Figs. 1 and 3), while the reference point for Pos2 coincides with the boundary region between Ap-g and Ap-h in Fig. 3.

Figure 5 shows that the kinematics in Mrk273 are disturbed at almost all locations in nuclear regions of the galaxies. For example, the narrow and intermediate components across Pos2 are well organised around the reference point $(D=0$ arc$\mathrm{sec})$. However, a third kinematic component emerges as we approach that point, and extends into the so-called N-Bubble region. Consistent with the results of RV13, we find extreme velocity shifts and widths of up to $\Delta V \sim-1000 \mathrm{~km} \mathrm{~s}^{-1}$ and $F W H M \sim 1400 \mathrm{~km} \mathrm{~s}^{-1}$, respectively, at these locations. In contrast, the emission line profiles along slit Pos 1 change drastically from one region to another. The kinematic properties of the narrowest component are relatively uniform across the region of $\sim 10$ arcsec that extends from Outflow-West to the prominent dust lane ${ }^{4}$. However, across this region, one or two additional kinematic components with substantially different widths and shifts are required to adequately model the emission line profiles.

Finally, Fig. 5 also shows the velocity shifts and widths of the ionised gas in the so-called extended filaments (Ap-e, Ap-f, Ap-i, Ap-j and Ap-k in Fig. 3). Interestingly, the emission lines at these locations show small velocity shifts and have the narrowest FWHM values $\left(38-111 \mathrm{~km} \mathrm{~s}^{-1}\right)$. In addition, the figure shows the presence of a positive velocity gradient across the filaments. This is further confirmed using the results from slit Pos3 and Pos4, which sample a larger fraction of the emission from the filaments observed in our ACS images.

Overall, Fig. 5 illustrates the complexity of the gas kinematics in Mrk273.

\subsection{Ionisation mechanisms}

\subsubsection{AGN and starburst photoionisation}

In this section, we investigate the nature of the ionising source(s) responsible for the emission lines observed at the different locations in Mrk273. Figure 6 shows the diagnostic line ratio diagrams (Baldwin et al. 1981; Veilleux \& Osterbrock 1987, hereafter BPT/VO87 diagrams) along with the optical classification scheme of Kewley et al. (2006). Overplotted on the figure are the results from our kinematic study. For this figure, we have used the extraction apertures shown in Table 3 and the eight apertures extracted for Slit Pos3 and Pos4. In addition we also used the Rodríguez Zaurín et al. (2009) WHT/ISIS spectrum of the source and modelled the emission lines for their nuclear extraction apertures (apertures D, E and F in their paper). Overall, a total of 22 apertures, which sample almost the entire nuclear emission and a large fraction of the emission at larger scales, were used to generate the plots in Fig. 6. The black triangles, red squares, and blue circles correspond to the narrow, intermediate, and broad components respectively. For clarity, no individual error bars are shown in the figure. However, to give an idea of the uncertainty associated with the line ratio measurements, a cross symbol indicates the median of all line ratio errors in each respective diagram. Individual error bars are shown later in the section, when a detailed analysis of some regions of interest is performed.

In the first place, it is notable that the line ratios measured at almost all locations in the galaxy are consistent with a Sy 2 classification, although a significant fraction of the points in Fig. 6 falls close to the Sy2/LINER limiting region. We find that the intermediate and broad components tend to have higher $[\mathrm{NII}] / \mathrm{H} \alpha$, $[\mathrm{OI}] / \mathrm{H} \alpha$, and $[\mathrm{SII}] / \mathrm{H} \alpha$ line ratios than the narrow components. Interestingly, such trend is not observed for the $[\mathrm{OIII}] / \mathrm{H} \beta$.

With the aim of better understanding the nature of ionisation mechanisms responsible for the measured line ratios, Fig. 6 also shows the Groves et al. (2004) dusty, radiation pressuredominated photoionisation models for NLR in AGN. For these models we assume a hydrogen density $n_{\mathrm{H}}=1000 \mathrm{~cm}^{-3}$ and an abundance that is four times greater than solar $\left(4 Z_{\odot}\right)$. This latter value was chosen based on the recent results of RZ13. These authors found that the line ratios derived for the ULIRGs in

4 Given the significant reddening effects, the kinematic results within the dust lane (i.e. the grey-shaded area) should be interpreted with caution. 

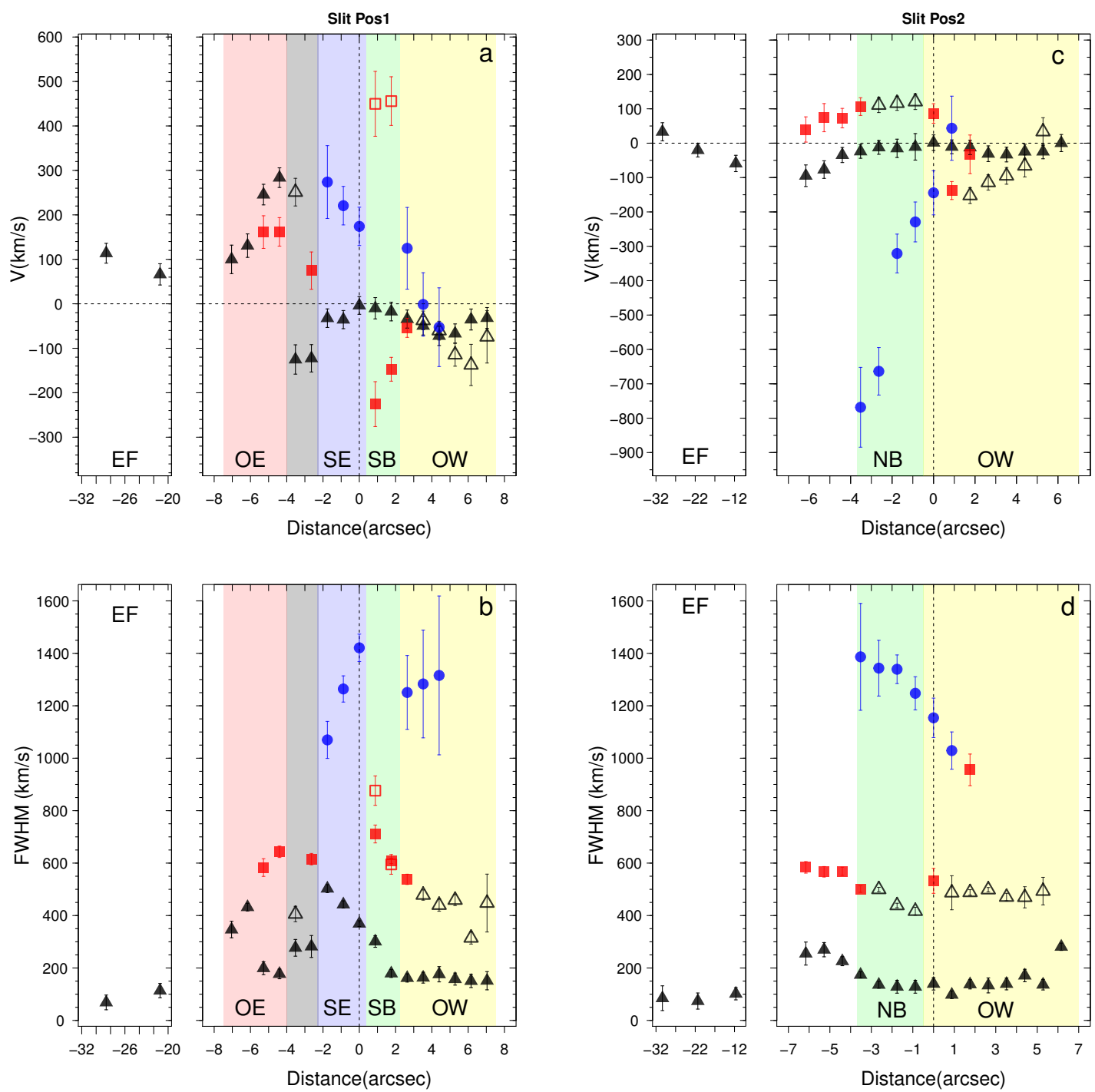

Fig. 5. Velocity shifts $(\Delta V)$ and widths (FWHM, corrected for instrumental width) plotted against the distance from a reference point in the galaxy, for Slit Pos1 and Pos2 (see the text for details on the selection $D=0$ arcsec reference point). Extraction apertures of 2 pixels (i.e. 0.88 arcsec) were used for the figure, except at the very extended regions, where we used the apertures Ap-e and Ap-f for Pos 1 and Ap-i, Ap-j and Ap-k for Pos2. Black triangles, red squares and blue circles correspond to the narrow, intermediate and broad components. To help the reader associate the points in the upper panels to those in the lower panels, when more than one kinematic component within the same FWHM range is present at a certain location, the second or third component (typically the broader) is indicated with an open symbol. For example, in Figs. 5a and 5b, the ionised gas emission at $D \sim 7$ arcsec is modelled using two kinematic components of widths $F W H M=152 \pm 25$ and $F W H M=456 \pm 106$, i.e. both within the narrow FWHM range. To be able to unambiguously associate the points in Fig. 5a to those in Fig. 5b at this location, the broader component is plotted with an open triangle. Finally, the shaded regions indicates the location in the galaxy. For Pos1, from west to east: yellow, green, blue and red correspond to Outflow-West (OW), S-Bubble (SB), SE compact structure and Outflow-East (OE) respectively. In addition, the grey-shaded region corresponds to the prominent dust lane observed to the east of the nuclear region. For Pos2, yellow and green correspond to Outflow-West and N-Bubble (NB) respectively. The region corresponding to the extended filaments (EF) is also indicated in the figure. (A color version of this figure is available in the online journal.)

their sample were consistent with gas of super-solar abundances $\left(4 Z_{\odot}\right)$ photoionised primarily by an AGN. A grid of models for various values of the ionising parameter $\left(U_{0}=0,-1,-2,-3\right.$, $-4)$ and ionising continuum SED power-law indicies $\left(F_{v} \propto v^{\alpha}\right.$, $\alpha=-1.2,-1.4,-1.7,-2.0)$ is shown in the figure.

In general, AGN photoionisation models reproduce the emission line ratios well in the sense that there is good consistency in the relative positions of the points and the models between the different diagrams. Interestingly, the biggest disagreement between the models and the observed line ratios occurs for the narrow kinematic components, for which the degeneracy issues mentioned in Sect. 3.2 are the least important (i.e. the flux associated with these components is better constrained). Finally, we have explored models with lower metallicities $\left(Z_{\odot}\right.$ and $\left.2 Z_{\odot}\right)$ and both higher and lower densities $\left(100\right.$ and $\left.10000 \mathrm{~cm}^{-3}\right)$, and find that the level of agreement not only does not improve, but it is worse.

\subsubsection{Shock ionisation}

As well as photoionisation by AGN and starburst, it is possible that line ratios plotted in Fig. 6 might be explained in terms of ionisation by fast radiative shocks (Dopita \& Sutherland 1995, 1996; Groves et al. 2004; Allen et al. 2008). Indeed, shocks are a plausible mechanism for accelerating the gas to the velocities 

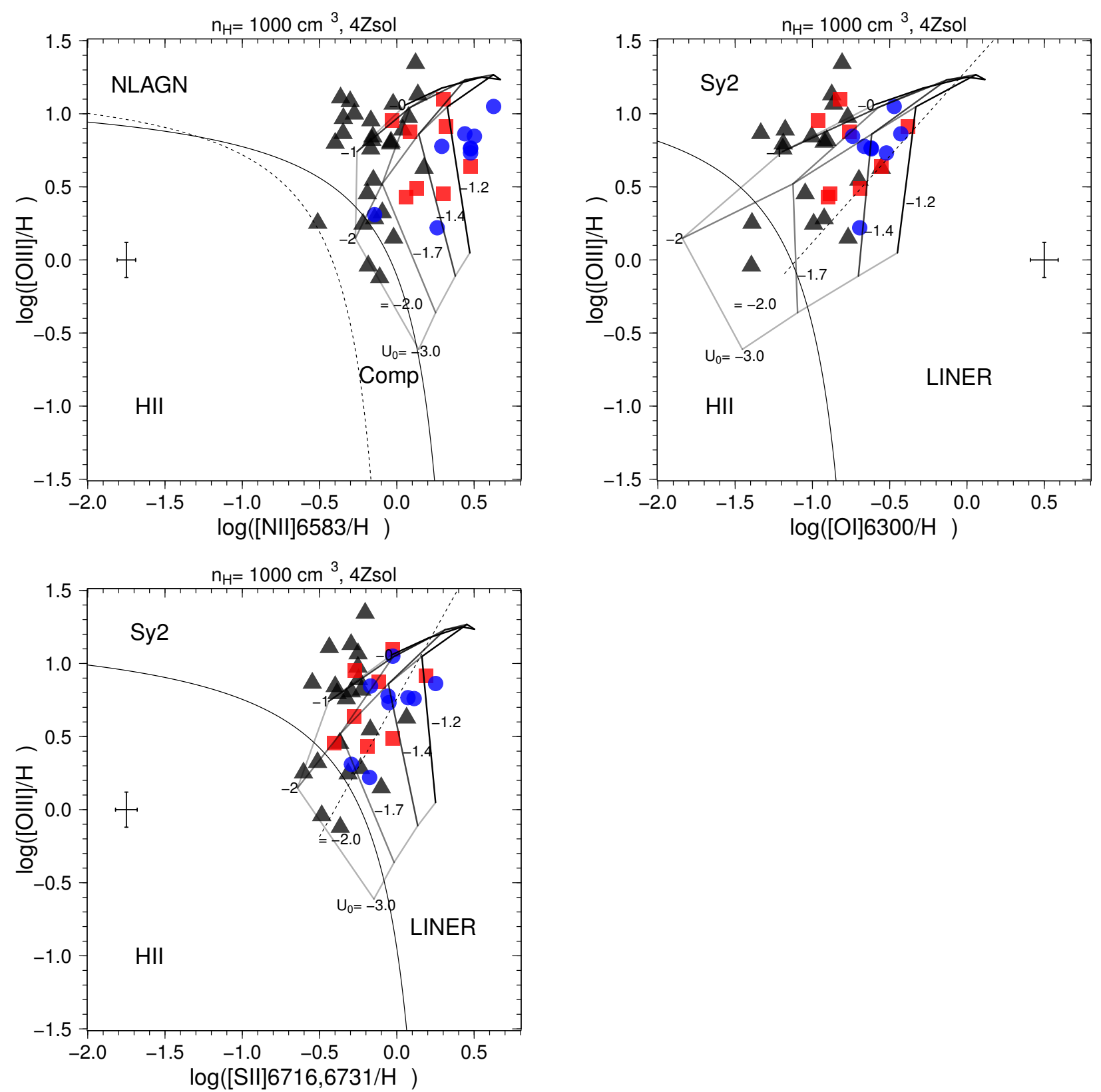

Fig. 6. Standard optical diagnostic diagrams showing the classification scheme of Kewley et al. (2006). The solid curves are the theoretical "maximum starburst line" derived by Kewley et al. (2001) as an upper limit for star-forming galaxies. The dashed curve in the [NII] diagram is the Kauffmann et al. (2003) semi-empirical lower boundary for the star forming galaxies, while the dashed lines in the [SII] and [OI] diagrams represent the empirical boundary lines between Seyfert 2 galaxies and LINERs. The Groves et al. (2004) grids of dusty, radiation pressuredominated models are also plotted in the figure. These grids have been generated assuming four times solar metallicity $\left(4 Z_{\odot}\right)$ and hydrogen density of $n_{\mathrm{H}}=1000 \mathrm{~cm}^{-3}$ (see the text for a justification on the selection of these parameters). Gridlines corresponding to five values of ionising parameter $\left(U_{0}=0,-1,-2,-3,-4\right)$ and four values of power law index $\left(F_{v} \propto v^{\alpha}, \alpha=-1.2,-1.4,-1.7,-2.0\right)$ are shown in the figure. To help the reader follow the gridlines these are grey-coded from "light-grey" to black, with light-grey and black corresponding to the lowest and highest values of $U_{0}$ and $\alpha$ respectively. Over-plotted on the diagrams are the results of our kinematic study. Black triangles are the line ratios corresponding to the narrow components, red squares correspond to the intermediate components and blue circles represent the broadest kinematic components. For clarity, no individual error bars are shown in the figure. However, to give an idea of the uncertainty associated with the line ratio measurements a cross symbol indicates the median of all line ratio errors in each respective diagram. (A color version of this figure is available in the online journal.)

that we measure in the nuclear regions of Mrk273. The shock models predict a series of line ratios for a range of magnetic field strengths $(B)$, electron densities $\left(n_{\mathrm{e}}\right)$, abundances, and shock velocities $\left(v_{\mathrm{s}}\right)$. In addition, the predicted line ratios depend on the geometry of the shock, including the presence or not of a photoionised precursor (see Allen et al. 2008, for a detailed discussion). In this context, Dopita \& Sutherland (1995) and Allen et al. (2008) found that the shock+precursor models produce a better fit to the line ratios measured for their samples of Sy2 galaxies. Furthermore, given the typical velocities associated with the ionised gas $\left(v>150 \mathrm{~km} \mathrm{~s}^{-1}\right)$, one would expect the photoionisation front to expand and form a precursor HII region ahead of the shock (see Dopita \& Sutherland 1995; Allen et al. 2008 , for a detailed discussion). For these reasons, we decided to use models with a precursor for the work presented in this section. 

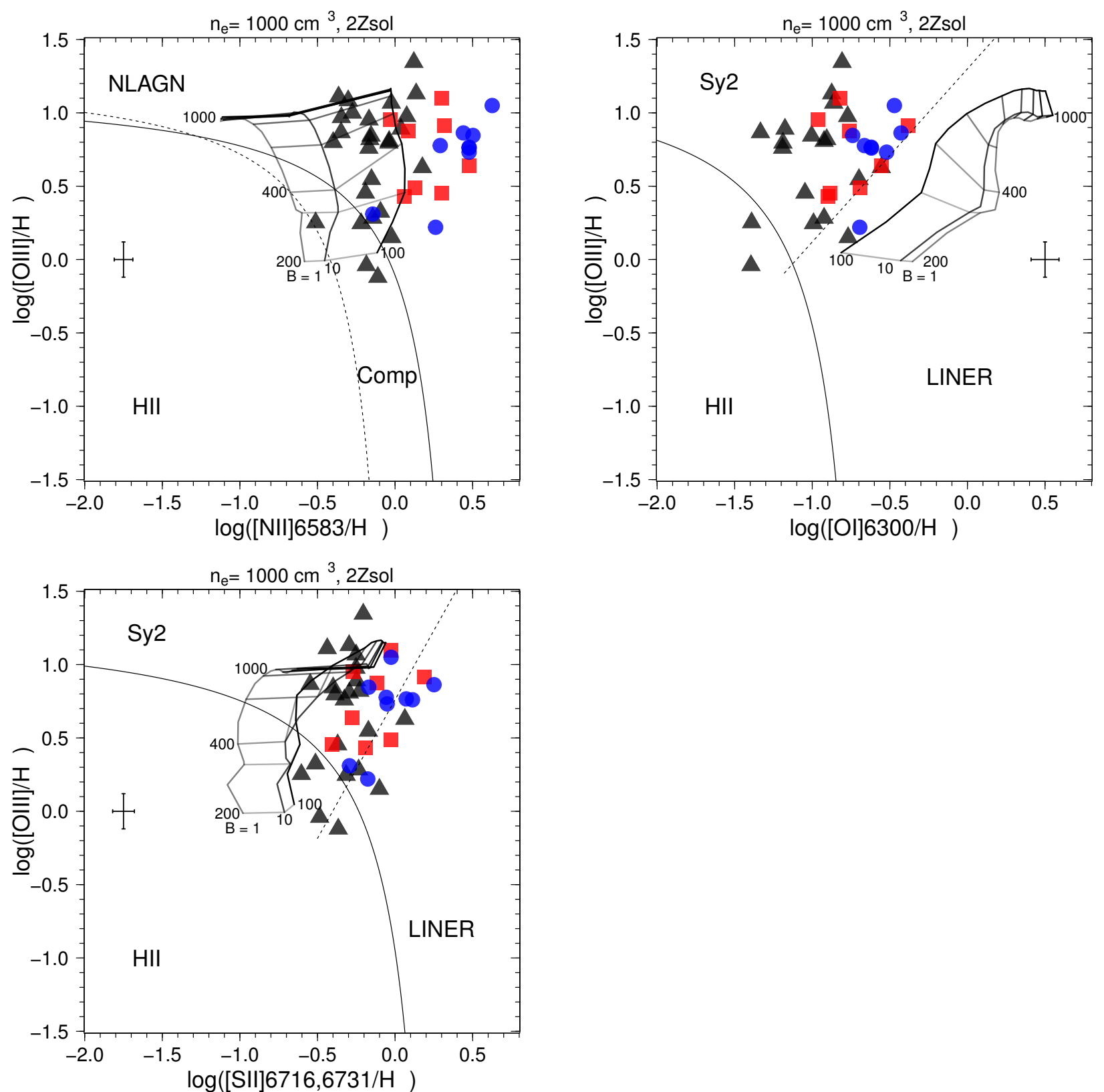

Fig. 7. Same as Fig. 6 but showing the Allen et al. (2008) grids of shock-ionisation models. These grids have been generated assuming a shock+precursor model with twice solar metallicity and pre-shock density of $n_{\mathrm{e}}=1000 \mathrm{~cm}^{-3}$. Gridlines corresponding to 3 values of magnetic field $(B=1,10$ and $100 \mu \mathrm{G})$ and 9 shock velocity values $\left(v_{\mathrm{s}}=200,300,400,500,600,700,800,900\right.$ and $\left.1000 \mathrm{~km} \mathrm{~s}^{-1}\right)$ are shown in the figure. $v_{\mathrm{s}}$ increases always from bottom to top with 3 values $\left(200,400\right.$ and $\left.1000 \mathrm{~km} \mathrm{~s}^{-1}\right)$ indicated in the figure. As in the case of Fig. 6, the gridlines these are grey-coded from "light-grey" to black, with light-grey and black corresponding to the lowest and highest values of $B$ and $v_{\mathrm{s}}$ respectively. The symbols and colors are the same as in Fig. 6. (A color version of this figure is available in the online journal.)

We note that RZ13 found that shock models generally fail to reproduce the emission line ratios measured in the nuclear regions of the galaxies in their sample of Sy-ULIRGs, unless some extreme parameter values are used (e.g. pre-shock density of $1000 \mathrm{~cm}^{-3}$ ). With this in mind, Fig. 7 shows the Allen et al. (2008) models with twice solar metallicity $\left(2 Z_{\odot}\right)$ and a pre-shock density of $1000 \mathrm{~cm}^{-3}$.

The value of the pre-shock density is one of the largest uncertainties in these models. For example, RZ13 carried out a spectroscopic study of a sample of Sy-ULIRGs and measured electron densities $\left(n_{\mathrm{e}}\right)$ of $\sim 1000 \mathrm{~cm}^{-3}$ (although $n_{\mathrm{e}}$ can be substantially higher in some extreme cases: see RZ13 for a discussion on the uncertainties associated with the electron density measurements). Assuming that these densities are associated with the compressed post-shock gas in the kinematically disturbed emission line components, then the pre-shock densities could be as low as $10-100 \mathrm{~cm}^{-3}$, since the compression factor in the cooled, post-shock gas can be high $(\sim 10-100$, see Dopita \& Sutherland 1995). We note that pre-shock densities of $1000 \mathrm{~cm}^{-3}$ would lead to post-shock densities of at least $10000 \mathrm{~cm}^{-3}$. Therefore, a pre-shock density of $1000 \mathrm{~cm}^{-3}$ is indeed an extreme assumption.

Even using the extreme pre-shock density of $1000 \mathrm{~cm}^{-3}$ (as assumed by RZ13), Fig. 7 shows that the majority of the measured line ratios do not fall within the region covered by the grids of shock models in the case of the $[\mathrm{OIII}] \lambda 5007 / \mathrm{H} \beta$ vs. 
[NII] $] 6583 / \mathrm{H} \alpha$. The disagreement is even higher for the other two diagrams, where practically none of the emission lines are covered by the gridlines. As in the previous section, we have exhaustively explored the parameter space $\left(B, v_{\mathrm{s}}, n_{\mathrm{e}}\right.$ and abundance). The agreement between the models and the measured emission lines improves when considering the extreme of $B=$ $1000 \mu \mathrm{G}$ (this is the highest $B$ value included in the Allen et al. 2008 models). However, such enormous magnetic fields are unlikely to be found in starburst galaxies (Thompson et al. 2006). Similarly, models with no precursor reproduce better the observed emission line ratios. However, as we mentioned before, given the high shock velocities indicated by the emission line kinematics, the presence of precursor HII regions ahead of the shock is expected.

Finally, it is possible to further test the importance of shock ionisation in Mrk273 by investigating whether there are correlations between the emission line kinematics and the line ratios. Figure 8 shows the line widths (FWHM) of the kinematic components at the different locations in the galaxy plotted against their corresponding line ratios. Shock models predict a strong correlation between these two quantities. Therefore, these plots have been used in the past to indicate the presence of shock ionisation (e.g. Armus et al. 1989; Dopita \& Sutherland 1995; Veilleux et al. 1995; Monreal-Ibero et al. 2006; Rich et al. 2011). In the case of Mrk273, we find that the FWHM and line ratios are significantly correlated, with a Spearman's rank correlation coefficient $(\rho)$ of $0.69,0.64$, and 0.63 for the $[\mathrm{NII}] / \mathrm{H} \alpha$, [OI] $/ \mathrm{H} \alpha$, and $[\mathrm{SII}] / \mathrm{H} \alpha$ plots respectively, and $p$-value $\ll 0.01$ for all three plots (assuming a t-distribution). However, as indicated by the $\rho$ values, the correlations are not particularly strong.

We further note that shocks may not be a unique explanation of any correlations between line width and line ratios. For example, it is possible that the emission lines in the kinematically disturbed regions were initially accelerated either by shocks or slow entrainment in an AGN-driven wind, but then photoionised by the AGN. In contrast, the more kinematically quiescent gas (with low FWHM) has not been accelerated and is part photoionised by stars or by the AGN at higher ionisation parameter (because it has a lower density due to the lack of shock acceleration). This combination of components could result in a correlation between line width and ionisation state, even if the more kinematically disturbed components are currently energised by the AGN photoinisation.

Overall, while the line ratios are generally more consistent with AGN photoionisation than they are with shocks, the correlations that we find between the line widths and the emission line ratios suggest that shocks may contribute at some level. Further work, using fainter diagnostic emission lines including, for example, [OIII] $\lambda 4363$ and HeII $\lambda 4686$, is required to determine the contribution of shocks to the line emission in the nuclear regions of Mrk273 in a definitive way.

\subsection{Mrk273: understanding the relation between the nuclear and extended ionised gas emission}

From the results presented above, it is clear that the morphology and kinematics of the warm ionised gas in Mrk273 are extremely complex. Disturbed emission line kinematics are observed at almost all locations around the nuclear regions. Our imaging and spectroscopic results suggest that we are witnessing a variety of phenomena occurring on different scales in the galaxy. To better understand these phenomena, we concentrate here in six apertures that sample the main regions of interest and are representative of the diverse kinematic properties observed. These are
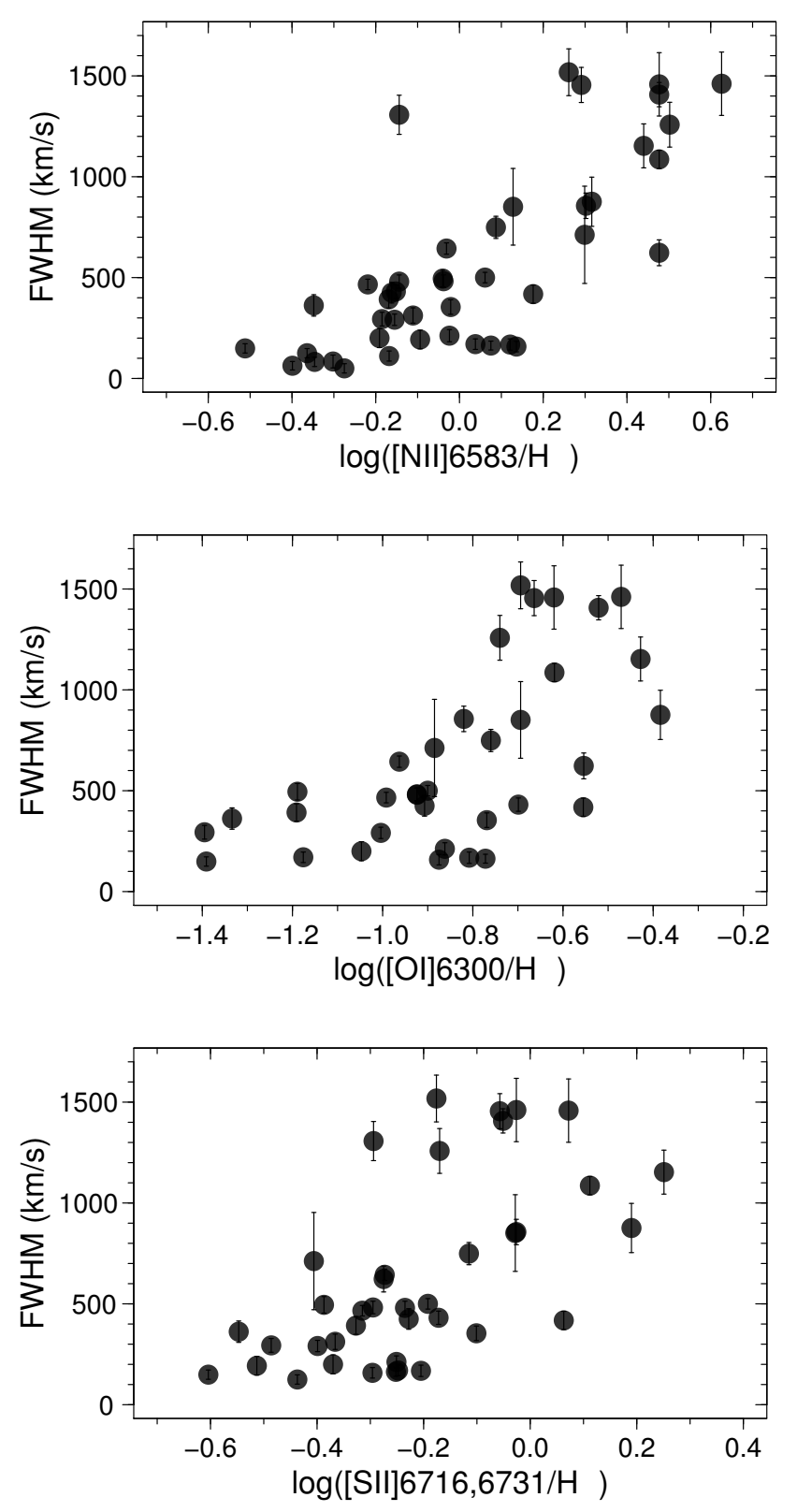

Fig. 8. Velocity widths (FWHM) of the kinematic components at the different locations in the galaxy plotted against the line ratio values.

Ap-a, Ap-b, Ap-c, Ap-d, Ap-h, and Ap-j (the modelling results for the $\mathrm{H} \alpha+[\mathrm{NII}]$ complex for these 6 apertures are shown in Fig. 4). Figure 9 shows the line ratios obtained for these particular apertures, while Table 4 shows the optical spectral type derived from each line ratio diagram and the adopted classification for each extraction aperture.

\subsubsection{The nuclear superbubble and the Outflow-West and Outflow-East structures}

In terms of the kinematics, our results are generally consistent with those of Colina et al. (1999) and RV13, and the kinematics in the central region of the galaxy can be described with a nuclear superbubble oriented N-S and a less collimated E-W outflow. However, the Colina et al. (1999) IFS observations have relatively low spatial and spectral resolution and the authors required only two kinematic components to model the emission 

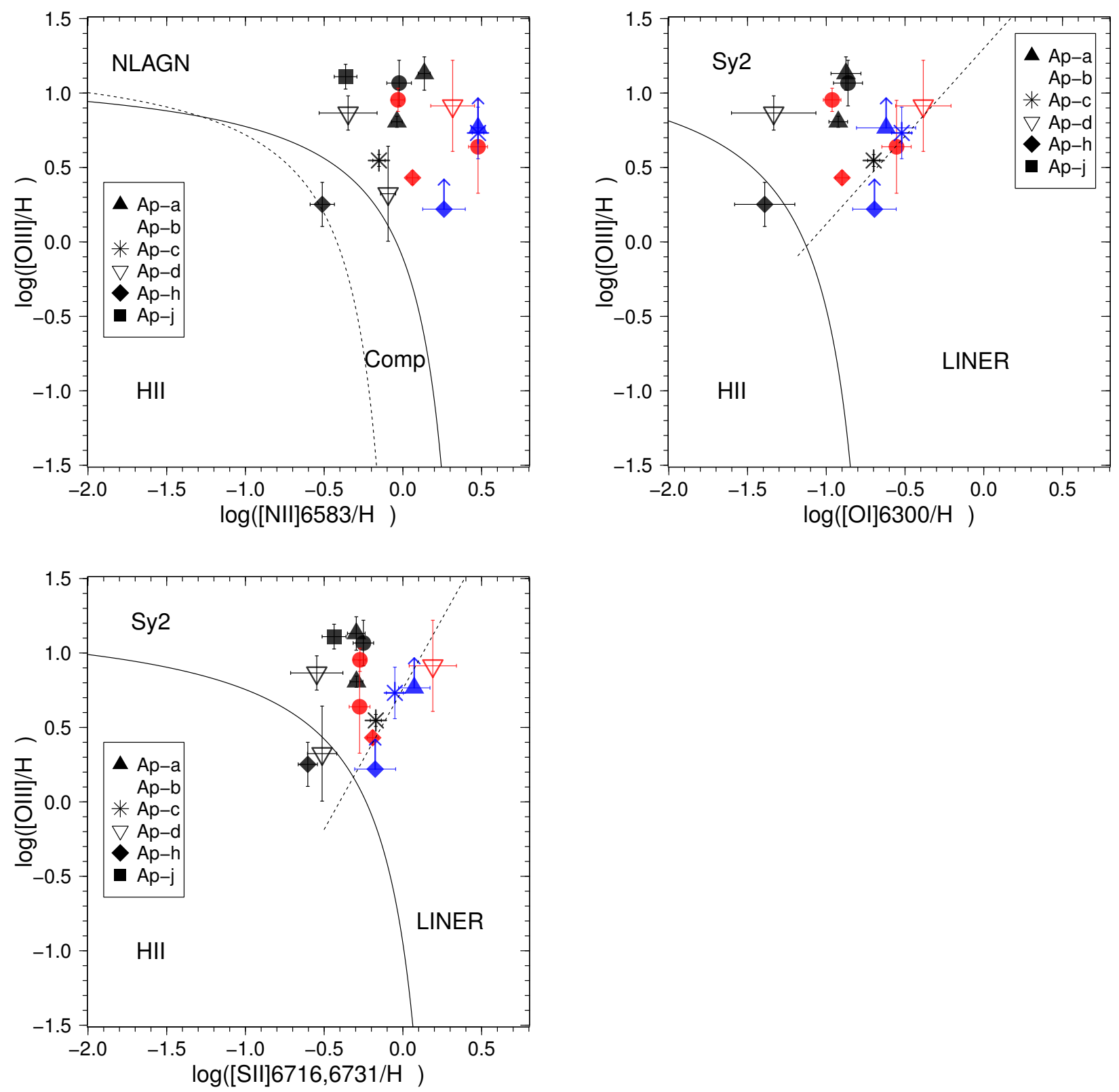

Fig. 9. Same as Fig. 6 but for the 6 apertures covering the main regions of interest in Mrk273 (see the text for details). The symbols correspond to the different apertures and are indicated in the figure, while the colors code the FWHM range: black, red and blue correspond to narrow, intermediate and broad kinematic components respectively. (A color version of this figure is available in the online journal.)

lines from the galaxy at all locations. On the other hand, the RV13 observations represent a significant improvement in sensitivity, spatial and spectral resolution with respect to those of Colina et al. (1999), although their FOV does not cover most of the region with enhanced [OIII] emission to the west and the entire [OIII] emission to the east of the nuclear region.

In this context, our new HST observations sample the entire emission from the galaxy at high spatial resolution, while the INT/IDS observations provide spectroscopic information for the nuclear and extended structures (albeit with lower spatial resolution). For example, the [OIII] morphology shown in Fig. 1, and the modelling results in Figs. 4 and 5 allow us to better constrain the properties of the E-W outflow. Our results suggest that Outflow-East and Outflow-West are part of the same outflow phenomenon, extending $\sim 4 \mathrm{kpc}$ from east to west at $\mathrm{PA} \sim 80^{\circ}$ relative to the RV13 nuclear superbubble.
In addition, covering a wavelength range of $\sim 4000-7200 \AA$, the INT/IDS observations allow us to study the ionisation mechanisms responsible for the emission at these locations. Figure 9 and Table 4 show that the line ratios at the location of the S-Bubble are consistent with Sy2 ionisation, while those corresponding to the location of N-Bubble show a mix between Sy2, LINER and HII ionisation in the different BPT/VO87 diagrams. As seen in Fig. 1, the S-Bubble is located closer to the SW nucleus and possibly illuminated by the AGN at that nucleus, which could explain the different line ratios relative to those of the N-Bubble.

In the case of Outflow-West region, line ratios consistent with Sy2 ionisation are found for all the 3 kinematic components in the three BPT/VO87 diagrams. This result, along with the location of the region relative to the SW nucleus, clearly suggest that this region is being illuminated by the AGN in the 
Table 4. Line ratios measured for the different apertures/regions and for the different kinematic components.

\begin{tabular}{|c|c|c|c|c|c|c|}
\hline \multirow{2}{*}{$\begin{array}{l}\text { Aperture } \\
\text { (1) }\end{array}$} & \multirow{2}{*}{$\begin{array}{l}\text { Region } \\
\text { (2) }\end{array}$} & \multirow{2}{*}{$\begin{array}{l}\text { Comp } \\
\text { (3) }\end{array}$} & \multicolumn{3}{|c|}{ BPT/VO87 } & \multirow{2}{*}{$\begin{array}{l}\text { Class } \\
\text { (7) }\end{array}$} \\
\hline & & & $\begin{array}{l}{[\mathrm{NII}] / \mathrm{H} \alpha} \\
\text { (4) }\end{array}$ & $\begin{array}{l}{[\mathrm{OI}] / \mathrm{H} \alpha} \\
(5)\end{array}$ & $\begin{array}{l}{[\mathrm{SII}] / \mathrm{H} \alpha} \\
\text { (6) }\end{array}$ & \\
\hline \multirow[t]{3}{*}{$\mathrm{Ap}-\mathrm{a}^{\star}$} & Outflow-W & N1 & S2 & S2 & $\mathrm{S} 2$ & S2 \\
\hline & & N2 & S2 & S2 & S2 & S2 \\
\hline & & B & S2 & S2 & $\mathrm{L} / \mathrm{S} 2$ & S2: \\
\hline \multirow[t]{3}{*}{ Ap-d } & Outflow-E & N1 & $\mathrm{S} 2 / \mathrm{H}$ & 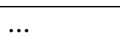 & $\mathrm{H} / \mathrm{S} 2$ & $\mathrm{Cp}$ \\
\hline & & $\mathrm{N} 2$ & S2 & S2 & S2 & $\mathrm{S} 2$ \\
\hline & & I & S2 & $\mathrm{S} 2 / \mathrm{L}$ & $\mathrm{L} / \mathrm{S} 2$ & $\mathrm{Cp}$ \\
\hline \multirow[t]{3}{*}{ Ap-b } & S-Bubble & $\mathrm{N}$ & S2 & S2 & S2 & S2 \\
\hline & & I1 & $\mathrm{S} 2$ & $\mathrm{~S} 2$ & $\mathrm{~S} 2$ & S2 \\
\hline & & I2 & S2 & S2/L & S2 & S2: \\
\hline \multirow[t]{3}{*}{ Ap-h ${ }^{\star}$} & N-Bubble & $\mathrm{N}$ & $\mathrm{H} /$ Comp & $\mathrm{H} / \mathrm{S} 2$ & $\overline{\mathrm{H}}$ & $\mathrm{Cp}$ \\
\hline & & I & S2 & $\mathrm{S} 2$ & $\mathrm{~S} 2 / \mathrm{L}$ & S2: \\
\hline & & B & S2 & $\mathrm{L} / \mathrm{S} 2$ & $\mathrm{~L} / \mathrm{S} 2$ & $\mathrm{Cp}$ \\
\hline \multirow[t]{2}{*}{ Ap-c } & SE compact & $\mathrm{N}$ & S2 & $\mathrm{S} 2 / \mathrm{L}$ & $\mathrm{S} 2 / \mathrm{L}$ & $\mathrm{Cp}$ \\
\hline & & B & S2 & $\mathrm{S} 2 / \mathrm{L}$ & $\mathrm{S} 2 / \mathrm{L}$ & $\mathrm{Cp}$ \\
\hline Ap-j & Ext. filaments & $\mathrm{N}$ & S2 & S2 & $\mathrm{S} 2$ & S2 \\
\hline
\end{tabular}

Notes. Column (1): extraction aperture. Column (2): corresponding region covered by the extraction aperture. Column (3): kinematic component. Columns (4)-(6): optical spectral type for each of the diagnostic diagrams. The symbols are: H = HII galaxies, L = LINER and S2 = Seyfert 2. Column (7): adopted optical spectral type. Cp indicates a mix between S2 and LINER and/or HII spectral types. Following Yuan et al. (2010) notation, single colons indicate apertures with the same spectral classification in two of the diagnostic diagrams. ${ }^{(\star)}$ For the broad component in these two apertures we assumed an $\mathrm{H} \alpha$ to $\mathrm{H} \beta$ ratio of 3. Therefore, the [OIII]/H$\beta$ line ratios calculated are lower limits of the real values.

SW nucleus of the galaxy. For Outflow-East, the line ratios show a mix of HII, LINER, and Sy2-like ionisation ${ }^{5}$.

\subsubsection{The SE compact structure}

Coinciding exactly with the location of the so-called SE nuclear component, we find the brightest and most compact $(F W H M=\sim 0.13$ arcsec or $\sim 100 \mathrm{pc}$, see Sect. 3.1) condensation of [OIII] emission in the galaxy. The nature of the SE component remains controversial. It was first identified as a star cluster based on NICMOS images (Scoville et al. 2000). Bondi et al. (2005) carried out a study of the source using milliarsecond resolution, high sensitivity radio observations. These authors found that the SE structure has a steep radio spectral index $(\alpha \simeq 1.4)$ with no compact and/or flat spectrum feature (Bondi et al. 2005), and is fully resolved at 5 mas resolution. However, despite the quality of their observations, the authors could not determine the nature of this nuclear component in a decisive way. On the other hand, U et al. (2013) carried out an AO-IFU study of the central kpc of the source. Their results based on the [SiVI] emission and the $[\mathrm{SiVI}] / \mathrm{Br} \alpha$ line ratios suggest that the dominant ionisation mechanisms for SE component is AGN photoionisation, although some contribution from shocks cannot be ruled out. Considering these results together with the morphology of the nuclear region observed in their IFU data, U et al. (2013) proposed a scenario in which the SE component is ionised by an AGN in the $\mathrm{N}$ nucleus that is completely obscured along our line of sight at optical wavelengths. This raises the possibility of Mrk273 being a dual AGN.

Figure 4 shows the $\mathrm{H} \alpha+[\mathrm{NII}]$ emission lines at the location of the SE component. Two kinematic components are required

\footnotetext{
5 The narrowest component $\left(F W H M=193 \pm 29 \mathrm{~km} \mathrm{~s}^{-1}\right)$ makes a negligible contribution to the [OI] emission within this extraction aperture (Ap-d). Therefore, this component is not required to adequately model the $[\mathrm{OI}]$ emission line profile (i.e., if included, its properties are entirely unconstrained). Hence, no $[\mathrm{OI}] / \mathrm{H} \alpha$ line ratio is shown in the corresponding line ratio diagram in Fig. 9.
}

to provide a good fit to the emission line profiles at this location: a narrow component with FWHM of $431 \pm 11 \mathrm{~km} \mathrm{~s}^{-1}$ and a broad component with $F W H M=1407 \pm 47 \mathrm{~km} \mathrm{~s}^{-1}$ redshifted $125 \pm 28 \mathrm{~km} \mathrm{~s}^{-1}$ relative to the narrow component. The line ratios for these two kinematic components fall in the limiting region between LINER and Sy2 ionisation and far from the HII ionisation region in the three BPT/VO87 diagrams.

To shed light on the nature of the SE compact structure, it is necessary to consider both the results in this paper and those of previous studies at other wavelengths. In the first place, we further emphasise that its structure is extremely compact, and it shows no morphological connection with the N or the SW nucleus in our HST images. In addition, it emits strong high ionisation emission lines at both optical (this study) and near-IR wavelengths (U et al. 2013). Finally, it is notable that a compact structure is also detected at this location in HST images taken with the $F 814 W$ filter (see Fig. 1 of U et al. 2013), which does not admit any strong emission lines for the redshift of Mrk273. This provides evidence that the SE component emits significant continuum emission at red-optical wavelengths, although the situation at near-IR wavelengths is less clear, because of the contamination of the $H$ - and $K$-band images by strong emission lines (U et al. 2013). Together, these results strongly suggest that the SE component is a separate nucleus with its own AGN rather than a patch of ISM illuminated by the AGN in one of the other nuclei (as suggested by U et al. 2013). In this scenario, the continuum emission detected from the SE component at red-optical wavelengths represents the direct light of stars in the remnant of the bulge surrounding the putative supermassive black hole.

The only caveat associated with this scenario is the lack of a clear X-ray or near-IR point source at the location of the SE component (Iwasawa et al. 2011). However, this can be explained if the X-ray AGN is heavily obscured by circumnuclear material, even at near-IR wavelengths, so that we are seeing the NLR of the AGN rather than the AGN continuum directly. It is also possible that the AGN at the SE nucleus has an intrinsically lower luminosity than the AGN in the SW (and N?) component. 
Perhaps, the NLR and any stellar component associated with the SE nucleus are less obscured that those of the other nuclei and, therefore, appear stronger at optical wavelengths.

Overall, our results are consistent with the idea that the SE component is a separate nucleus with a strongly obscured and, perhaps, relatively low luminosity, AGN. However, there are some caveats associated with this scenario, and more observations are required to help elucidate the nature of this compact structure.

\subsubsection{The extended filaments}

The most spectacular features observed in the HST-[OIII] and the INT-H $\alpha$ images of the galaxy are the filaments and clumps of ionised gas emission extended $\sim 30 \operatorname{arcsec}(\sim 23 \mathrm{kpc})$ to the east of the nuclear region. Interestingly, the kinematics at all locations in these filaments covered by our slits are drastically different from those in the nuclear regions, with no signs of multiple kinematic components. In fact, a kinematic model comprising a very narrow component $\left(F W H M \lesssim 100 \mathrm{~km} \mathrm{~s}^{-1}\right)$ is sufficient to model the emission lines from the extended filaments. In addition, our kinematic results suggest the presence of a positive velocity gradient across the structure, with velocity shifts increasing from $\Delta V$ of $-77 \pm 24 \mathrm{~km} \mathrm{~s}^{-1}$ relative to the rest frame in the inner regions to $114 \pm 27 \mathrm{~km} \mathrm{~s}^{-1}$ in the outer regions.

Regarding the ionisation mechanisms, for all locations across the extended filaments the line ratios fall within the Sy 2 ionisation region of the three BPT/VO87 diagrams shown in Fig. 9. We note that the degeneracy issues do not affect the modelling results in this case, since only one kinematic component is required to model each emission line. In addition, the continuum emission associated with this structure is relatively weak. Therefore, the line ratios measured for the ionised gas emission in the extended filaments and clumps are extremely reliable.

It is interesting to consider how this extended structure might have formed. In the first place, it is well known that major galaxy mergers are violent events, with a duration of up to a few Gyrs from the first encounter to the time when the two nuclei finally coalesce (e.g. Mihos \& Hernquist 1996; Barnes 2004; Springel et al. 2005; Cox et al. 2008; Johansson et al. 2009). At various stages during the merger event, numerical simulations show that the gas rains down into the central regions of the merging galaxies triggering star formation, and possibly, AGN activity (e.g. Springel et al. 2005; Cox et al. 2006; Hopkins \& Elvis 2010). This generates outflows that intermittently blow out material from the central regions to larger scales (e.g. Hopkins et al. 2005; Springel et al. 2005; Cox et al. 2006). As the merger event progresses, the high expansion velocity of the material ejected at early stages gradually decreases due to gravitational effects. Part of this material eventually escapes from the potential well of the merging system into the IGM, while the fraction that remains gravitationally bound is accreted again onto the central region.

In this context, Mrk273 is a merger in its late stages, where the two (or three) nuclei are close to coalescence. The [OIII] emission from the extended structure shows a bubble-like morphology that, at first sight, suggests that the gas has been blown out from the nuclear region. Therefore, it is possible that the emission in the extended filaments is associated with an outflow induced by AGN-activity at early stages in the merger. In this scenario, the gas in the filaments has progressively slowed down and any turbulence has partially dissipated, which would partially explain the emission line properties at these locations.

Another possibility is that the extended filaments are related to a more recent outflow event and the gas in the filaments is outflowing at high velocities in a direction that is almost perpendicular to the line of sight. In this case, the quiescent kinematics of the extended emission would be explained in terms of projection effects. However, if the ionised gas emission in the extended filaments is really due to outflows (either induced by past AGN activity or related with a more recent event), one would expect the emission lines from the extended filaments to show at least some evidence for broadening due to turbulence in the outflow. As described before, we find no evidence for complex kinematics, and the ionised gas in the extended filament is extremely quiescent (i.e. very narrow FWHM). Certainly these results do not favour a scenario in which the gas in the extended filaments is related to an outflow event.

Furthermore, our continuum image, as well as previous broad-band continuum (or continuum dominated) images from the ground (Mazzarella \& Boroson 1993; Hibbard \& Yun 1996; Kim et al. 2002) and the HST (GO 10592, PI: Aaron Evans) show significant continuum emission at the locations of the extended filaments. Such emission is likely to trace the starlight. However, it seems unlikely that stars would be able to form in the extreme conditions of an outflow (but see Tadhunter et al. 2014; Zubovas \& King 2014). Therefore, considering all these results, we favour a scenario in which the entire ionised gas emission from the filaments and clumps represents tidal debris left over from a secondary merger event that is illuminated by one of the AGNs in the galaxy. This would be consistent with the quiescent kinematics of the extended filaments, the Sy2 ionisation line ratios, and also the presence of continuum emission, which could represent the emission from stars associated with one of precursor galaxies that now form part of the tidal debris. We note that this scenario resembles that described by McDowell et al. (2003) for Arp220. In that paper, the authors found that "lobes" of extended, faint, edge-brightened ionised gas emission were likely related to the merger dynamics rather than the result of an outflow. Therefore, it is possible that such low surface brightness features are common among these types of merger remnants, but have so far been difficult to detect.

In terms of the relationship between the extended filaments and the nuclear structures, the morphology of the emission from the filaments closer to the nuclear region suggest that they emerge from the $\mathrm{N}$ nucleus. This is an interesting result, since, as we mentioned before, the line ratios at all locations in the filaments are consistent with Sy2 ionisation. However, currently there is no direct evidence for the presence of an AGN in that nucleus. It is also worth remembering that the $\mathrm{N}$ nucleus is embedded in a massive rotating disk of molecular gas with a major axis aligned along PA $=70^{\circ}-90^{\circ}$ (Downes \& Solomon 1998; Cole et al. 1999). Therefore, it is possible that the AGN in that nucleus is too heavily obscured to be detected in the current optical and infrared observations of the source. In addition, it is unlikely that any putative AGN within that disk illuminates the entire [OIII] nebula, as the disk would absorb the radiation along the major axis. In this context, it is possible that the extended filaments are tidal debris from the galaxy that hosted the $\mathrm{N}$ or the SE nucleus, but are illuminated by the AGN in the SW (or the SE) component. A second possibility is that the AGN in the $\mathrm{N}$ nucleus has temporarily switched off so that the extended emission from the filaments represents a "light echo", reflecting a previous phase of AGN activity.

Clearly, further observations are required to investigate the origin of the extended filamentary structure and its relationship with the nuclear structures. For example, large FOV, deep IFS observations covering the full extent of the filaments would help to distinguish between the possible scenarios described in 
this section for the origin of this structure. In addition, they would be extremely useful to investigate in detail which of the nuclear structures is directly related to the extended filaments.

\section{Summary}

We have used HST/ACS and INT/WFC observations to carry out an optical imaging and spectroscopic study of the warm, ionised gas in the nuclear and extended regions of Mrk273. The results from this study show that the emission line kinematics in this galaxy are extremely complex, with multiple outflows occurring in the nuclear regions of the galaxy out to a radial distance of $4 \mathrm{kpc}$ from the $\mathrm{N}$ nucleus. In addition, we find emission line ratios that are consistent with AGN photoionisation as the dominant ionising mechanism at most locations in the galaxy sampled by our slit. Shocks may also be present, but our results suggest that they are not the dominant source of ionisation. To gain a better idea of the physical processes ongoing in Mrk273, we have concentrated on six extraction apertures that sample the main regions of interest and have investigated their kinematics and ionisation mechanisms in detail. The results can be summarised as follows.

- The nuclear superbubbles: the presence of nuclear superbubbles oriented N-S was reported by RV13. Our HST [OIII] image of the galaxy shows a U-shaped structure to the north of the nuclear region that traces the northern bubble (N-Bubble) well. The southern bubble (S-Bubble) is not visible in our ACS image; this can be explained in terms of reddening effects. The kinematics measured at the locations of the bubbles are consistent with those measured by RV13 and are the most extreme in the galaxy. The line ratios indicate a mix between HII, LINER, and Sy2 mechanisms for N-Bubble, while S-Bubble has line ratios typical of Sy2 ionisation for all the kinematic components. These results are consistent with the nuclear superbubbles emerging from the $\mathrm{N}$ nucleus of the galaxy. The proximity of S-Bubble to the SW nucleus could explain the different line ratios between the two sides of the bubbles. Of course, another possibility is that the line ratios in S-Bubble are directly related to an AGN buried in the $\mathrm{N}$ nucleus.

- Outflow-West and Outflow-East: three kinematic components are required to model the emission from both OutflowWest and Outflow-East. In the case of Outflow-West, emission line ratios consistent with Sy2 ionisation are found for the three kinematic components in the three BPT/VO87 diagrams. These results, along with the location of the OutflowWest in the galaxy clearly suggest that this region is being illuminated by the AGN in the SW nucleus. For OutflowEast the line ratios show a mix between HII, LINER, and dominant Sy2 ionisation. Our results suggest that OutflowEast is related to Outflow-West and that the two structures correspond to a nuclear outflow expanding from west to east almost perpendicular to the nuclear superbubbles.

- The SE compact structure: coinciding with the location of the so-called SE nuclear component, this is the brightest and most compact structure observed in the HST [OIII] image of the galaxy. The compact nature of this structure, the high ionisation state derived from our optical study, and the near-IR study of U et al. (2013), and the presence of significant continuum emission at this location at red optical wavelengths together strongly suggest that the SE component is a separate nucleus with its own AGN rather than a clump of ionised gas illuminated by one of the other AGNs, as proposed by U et al. (2013).

- The extended filaments: the ACS-[OIII] and INT-H $\alpha$ images of the galaxy show a spectacular system of filaments and knots of emission extending $\sim 23 \mathrm{kpc}$ to the east of the nuclear region. A kinematic model comprising a single, narrow Gaussian component provides a good fit to the emission lines, and the lines ratios are consistent with Sy2 ionisation, at all locations in the filament system. These results suggest that the gas emission in the filaments is associated with tidal debris left over from a secondary merger event, with the filaments ionised by an AGN in the nuclear regions of the galaxy. Indeed, it is possible that Outflow-West, OutflowEast and the extended filaments represent ionisation cones emerging from one of the nuclei.

Mrk273 is a nearby ULIRG with a variety of interesting phenomena occurring at all scales in the galaxy. Therefore, it offers a perfect opportunity to investigate the physical processes that govern the relationship between the nuclear activity and the surrounding ISM in gas-rich mergers. The new HST images reveal a very disturbed morphology in the nuclear region of the galaxy, along with a spectacular filamentary structure extending $\sim 23 \mathrm{kpc}$ to the east, whose origin remains uncertain. In addition, we have identified the brightest and the most compact structure in [OIII] emission with the SE component. Our results suggest that this component is a separate nucleus with its own AGN. If this result is confirmed, as well as those of U et al. (2013), which suggest the presence of an AGN in the N disk, three AGN could potentially be co-existing in the nuclear region of Mrk273.

Overall, these results demonstrate the potential of deep observations of the extended emission line gas for unravelling the complexity of ULIRGs, determining the nature of triggering mergers, and gauging the importance of feedback effects associated with their AGN.

Acknowledgements. J.R.Z. and C.R.A. acknowledge financial support from the spanish grant AYA2010_21887-C04-04. The Isaac Newton Telescope is operated on the island of La Palma by the Isaac Newton Group in the Spanish Observatorio del Roque de los Muchachos of the Instituto de Astrofisica de Canarias. J.R.Z. would like to thank Manuel Díaz Alfaro and Javier Piqueras López for the help with the R and IDL codes used in this paper. This research is supported in part by STFC grant St/J001589/1 and a Marie Curie IEF within the 7th European community framework programme (PIEF-GA-2012-327934).

\section{References}

Allen, M. G., Groves, B. A., Dopita, M. A., Sutherland, R. S., \& Kewley, L. J. 2008, ApJS, 178, 20

Armus, L., Heckman, T. M., \& Miley, G. K. 1989, ApJ, 347, 727

Armus, L., Charmandaris, V., Bernard-Salas, J., et al. 2007, ApJ, 656, 148

Baldwin, J. A., Phillips, M. M., \& Terlevich, R. 1981, PASP, 93, 5

Barber, C. R., Roberts, T. P., \& Warwick, R. S. 1996, MNRAS, 282, 157

Barnes, J. 2004, MNRAS, 350, 798

Bedregal, A. G., Colina, L., Alonso-Herrero, A., \& Arribas, S. 2009, ApJ, 698, 1852

Benson, A. J., Bower, R. G., Frenk, C. S., et al. 2003, ApJ, 599, 38

Bondi, M., Pérez-Torres, M.-A., Dallacasa, D., \& Muxlow, T. W. B. 2005, MNRAS, 361, 748

Carilli, C., \& Taylor, G. 2000, ApJ, 532, L95

Cicone, C., Feruglio, C., Maiolino, R., et al. 2012, A\&A, 543, A99

Cicone, C., Maiolino, R., Sturm, E., et al. 2014, A\&A, 562, A21

Clements, D. L., Sutherland, W. J., McMahon, R. G., \& Saunders, W. 1996, MNRAS, 279, 477

Cole, G., Pedlar, A., Holloway, A., \& Mundell, C. 1999, MNRAS, 310, 1033

Colina, L., Arribas, S., \& Borne, K. D. 1999, ApJ, 527, L13

Condon, J., Huang, Z.-P., Yin, Q., \& Thuan, T. 1991, ApJ, 378, 65

Cox, T. J., Jonsson, P., Primack, J. R., \& Somerville, R. S. 2006, MNRAS, 373, 1013 
Cox, T. J., Jonsson, P., Somerville, R. S., Primack, J. R., \& Dekel, A. 2008, MNRAS, 384, 386

di Matteo, T., Springel, V., \& Hernquist, L. 2005, Nature, 433, 604

Dopita, M. A., \& Sutherland, R. S. 1995, ApJ, 455, 468

Dopita, M. A., \& Sutherland, R. S. 1996, ApJS, 102, 161

Downes, D., \& Solomon, P. 1998, ApJ, 507, 615

Fabian, A. C. 1999, MNRAS, 308, L39

Feruglio, C., Maiolino, R., Piconcelli, E., et al. 2010, A\&A, 518, L155

Fischer, J., Sturm, E., González-Alfonso, E., et al. 2010, A\&A, 518, L41

Genzel, R. Lutz, D., Sturm, E., Egami, E., et al. 1998, ApJ, 498, 579

Groves, B. A., Dopita, M. A., \& Sutherland, R. S. 2004, ApJS, 153, 9

Hibbard, J. E., \& Yun, M. S. 1996, in Cold Gas at High Redshift, eds. M. N. Bremer, \& N. Malcolm, Astrophys. Space Sci. Lib., 206, 47

Hopkins, P. F., \& Elvis, M. 2010, MNRAS, 401, 7

Hopkins, P. F., Hernquist, L., Cox, T. J., et al. 2005, ApJ, 630, 705

Howell, J. H., Armus, L., Mazzarella, J. M., et al. 2010, ApJ, 715, 572

Iwasawa, K., Mazzarella, J. M., Surace, J. A., et al. 2011, A\&A, 528, A137

Johansson, P. H., Burkert, A., \& Naab, T. 2009, ApJ, 707, L184

Kauffmann, G., Heckman, T. M., Tremonti, C., et al. 2003, MNRAS, 346, 1055

Kewley, L. J., Heisler, C. A., Dopita, M. A., \& Lumsden, S. 2001, ApJS, 132, 37

Kewley, L. J., Groves, B., Kauffmann, G., \& Heckman, T. 2006, MNRAS, 372, 961

Kim, D. C., Veilleux, S., \& Sanders, D. B. 2002, ApJ, 143, 277

Knapen, J., Laine, S., Yates, J. A., et al. 1997, ApJ, 490, L29

Majewski, S., Hereld, M., Koo, D., Illingworth, G., \& Heckman, T. 1993, ApJ, 403, 125

Markwardt, C. B. 2009, in Astronomical Data Analysis Software and Systems XVIII, eds. D. A. Bohlender, D. Durand, \& P. Dowler, ASP Conf. Ser., 411, 251

Mazzarella, J. M., \& Boroson, T. A. 1993, ApJS, 85, 27

McDowell, J. C., Clements, D. L., Lamb, S. A., et al. 2003, ApJ, 591, 154

Mihos, J., \& Hernquist, L. 1996, ApJ, 464, 641

Monreal-Ibero, A., Arribas, S., \& Colina, L. 2006, ApJ, 637, 138

Murphy, Jr., T. W., Armus, L., Matthews, K., et al. 1996, AJ, 111, 1025
Osterbrock, D., Fulbright, J., Keane, M., \& Trager, S. 1996, PASP, 108, 277 Rich, J. A., Kewley, L. J., \& Dopita, M. A. 2011, ApJ, 734, 87

Rodríguez Zaurín, J., Tadhunter, C. N., \& González Delgado, R. M. 2009, MNRAS, 400, 1139

Rodríguez Zaurín, J., Tadhunter, C. N., \& González Delgado, R. M. 2010 MNRAS, 403, 1317

Rodríguez Zaurín, J., Tadhunter, C. N., Rose, M., \& Holt, J. 2013, MNRAS, 432, 138

Rupke, D. S. N., \& Veilleux, S. 2011, ApJ, 729, L27

Rupke, D. S. N., \& Veilleux, S. 2013a, ApJ, 775, L15

Rupke, D. S. N., \& Veilleux, S. 2013b, ApJ, 768, 75

Sanders, D. B., \& Mirabel, I. F. 1996, ARA\&A, 34, 749

Scoville, N. Z., Evans, A. S., Thompson, R., et al. 2000, ApJ, 119, 991

Silk, J., \& Rees, M. J. 1998, A\&A, 331, L1

Soifer, B., Neugebauer, G., Matthews, K., et al. 2000, AJ, 119, 509

Spoon, H. W. W., Farrah, D., Lebouteiller, V., et al. 2013, ApJ, 775, 127

Springel, V., Di Mateo, T., \& Hernquist, L. 2005, MNRAS, 361, 776

Sturm, E., González-Alfonso, E., Veilleux, S., et al. 2011, ApJ, 733, L16

Surace, J. A., \& Sanders, D. B. 2000, AJ, 120, 604

Surace, J. A., Sanders, D. B., \& Evans, A. S. 2000, ApJ, 529, 170

Tadhunter, C., Morganti, R., Rose, M., Oonk, J. B. R., \& Oosterloo, T. 2014, Nature, 511, 440

Thompson, T. A., Quataert, E., Waxman, E., Murray, N., \& Martin, C. L. 2006, ApJ, 645, 186

U, V., Medling, A., Sanders, D., et al. 2013, ApJ, 775, 115

Veilleux, S., \& Osterbrock, D. E. 1987, ApJS, 63, 295

Veilleux, S., Kim, D. C., Mazzarella, J. M., \& Soifer, B. T. 1995, ApJ, 98, 171

Veilleux, S., Kim, D. C., \& Sanders, D. B. 2002, ApJ, 143, 315

Veilleux, S., Rupke, D. S. N., Kim, D.-C., et al. 2009, ApJS, 182, 628

Veilleux, S., Melendez, M., Sturm, E., et al. 2013, ApJ, in press [arXiv: 1308.3139]

Westmoquette, M. S., Clements, D. L., Bendo, G. J., \& Khan, S. A. 2012, MNRAS, 424, 416

Yuan, T.-T., Kewley, L. J., \& Sanders, D. B. 2010, ApJ, 709, 884

Zubovas, K., \& King, A. 2014, MNRAS, 439, 400 